\title{
MicroRNA-31 initiates lung tumorigenesis and promotes mutant KRAS-driven lung cancer
}

\author{
Mick D. Edmonds, ${ }^{1}$ Kelli L. Boyd, ${ }^{1}$ Tamara Moyo, ${ }^{2}$ Ramkrishna Mitra, ${ }^{3}$ Robert Duszynski, ${ }^{1}$ Maria Pia Arrate, ${ }^{1}$ \\ Xi Chen, ${ }^{4}$ Zhongming Zhao, ${ }^{3,5}$ Timothy S. Blackwell, ${ }^{2}$ Thomas Andl, ${ }^{2}$ and Christine M. Eischen ${ }^{1}$ \\ 'Department of Pathology, Microbiology and Immunology, ${ }^{2}$ Department of Medicine, and ${ }^{3}$ Department of Biomedical Informatics, Vanderbilt University Medical Center, Nashville, Tennessee, USA. \\ ${ }^{4}$ Department of Public Health Sciences and Sylvester Comprehensive Cancer Center, University of Miami Miller School of Medicine, Coral Gables, Florida, USA. ${ }^{5}$ Center for Quantitative Sciences, \\ Vanderbilt University Medical Center, Nashville, Tennessee, USA.
}

\begin{abstract}
MicroRNA (miR) are important regulators of gene expression, and aberrant miR expression has been linked to oncogenesis; however, little is understood about their contribution to lung tumorigenesis. Here, we determined that miR-31 is overexpressed in human lung adenocarcinoma and this overexpression independently correlates with decreased patient survival. We developed a transgenic mouse model that allows for lung-specific expression of miR-31 to test the oncogenic potential of miR-31 in the lung. Using this model, we observed that miR-31 induction results in lung hyperplasia, followed by adenoma formation and later adenocarcinoma development. Moreover, induced expression of miR-31 in mice cooperated with mutant KRAS to accelerate lung tumorigenesis. We determined that miR-31 regulates lung epithelial cell growth and identified 6 negative regulators of RAS/MAPK signaling as direct targets of miR-31. Our study distinguishes miR-31 as a driver of lung tumorigenesis that promotes mutant KRAS-mediated oncogenesis and reveals that miR-31 directly targets and reduces expression of negative regulators of RAS/MAPK signaling.
\end{abstract}

\section{Introduction}

Lung cancer is the leading cause of cancer-associated deaths worldwide, and adenocarcinoma is the predominant subtype of non-small-cell lung cancer (NSCLC) (1). The processes necessary for the initiation and progression of lung tumorigenesis require vast changes in gene regulation. microRNA ( $\mathrm{miR}$ ) are noncoding RNA ( $22 \mathrm{nt})$ that can regulate the expression of mRNA, typically by binding 3 '-untranslated regions (3'-UTRs), leading to decreased translation and/or degradation of the target mRNA (2). Thus, miR are likely well suited to facilitate the many changes required for cellular transformation. However, there is a paucity of functional data demonstrating this in the lung.

Despite their dysregulation being reported in nearly every human malignancy, only a handful of miR have been demonstrated to elicit the changes required for the initiation of tumorigenesis, and no studies in this regard have been performed in lung (3-5). miR-21, which is considered an oncomiR, as it is overexpressed in many human cancers and has oncogenic function in vitro, was insufficient to initiate lung tumorigenesis in mice. However, miR-21 was able to enhance mutant KRAS-driven lung tumor growth in vivo (6). KRAS is a $G$ protein that activates the MAPK signaling cascade, resulting in cell growth and cell survival. Point mutations that lead to the constitutive activation of KRAS drive events in multiple cancers, including approximately one-third of human lung adenocarcinoma $(7,8)$. However, RAS/MAPK pathway activation is reported in $76 \%$ of lung adenocarcinoma (8), suggesting that other mechanisms of RAS/MAPK pathway activation, possibly by miR, contribute to this malignancy.

Conflict of interest: The authors have declared that no conflict of interest exists. Submitted: May 8, 2015; Accepted: November 6, 2015.

Reference information: / Clin Invest. 2016;126(1):349-364. doi:10.1172/JCI82720.
Many miR appear to have tissue-specific functions, including miR-31, which adds complexity to the study of miR. miR-31 is reported to have tumor-suppressing functions, and its expression is decreased during tumorigenesis in several types of cancer, including breast, ovarian, prostate, bladder, hepatocellular, and gastric carcinoma and melanoma (9-16). In contrast, a proproliferative function for miR-31 was reported in colorectal, head and neck squamous cell, small-cell lung, and esophageal squamous cell carcinoma $(13,17-20)$. In the lung, miR-31 overexpression was observed in lung cancers from cyclin E transgenic mice and in 2 of 3 human lung adenocarcinomas and all 3 lung squamous cell carcinomas analyzed (21). Inhibition of miR-31 resulted in reduced numbers of lung adenocarcinoma cells (21), whereas elevated miR-31 expression was shown to correlate with lymph node metastasis in patients with lung adenocarcinoma (22). Despite these reports, the contribution and mechanism of miR-31 function in lung adenocarcinoma remain unclear. To address this, we evaluated miR-31 expression in patient cohorts and generated a mouse model to assess the contribution of miR-31 to lung tumorigenesis. We determined that miR-31 was highly overexpressed in lung adenocarcinoma and significantly correlated with patient survival. miR-31 had a proproliferative function in both transformed and nontransformed lung epithelial cells and initiated tumorigenesis and promoted mutant KRAS-driven lung cancer in vivo. Notably, we determined that miR-31 directly targeted multiple negative regulators of RAS/MAPK signaling, leading to increased signaling from this pathway. Taken together, our data indicate that miR31 is an initiator of lung tumor development and promoter of lung adenocarcinoma progression through regulation of RAS/ MAPK signaling. 

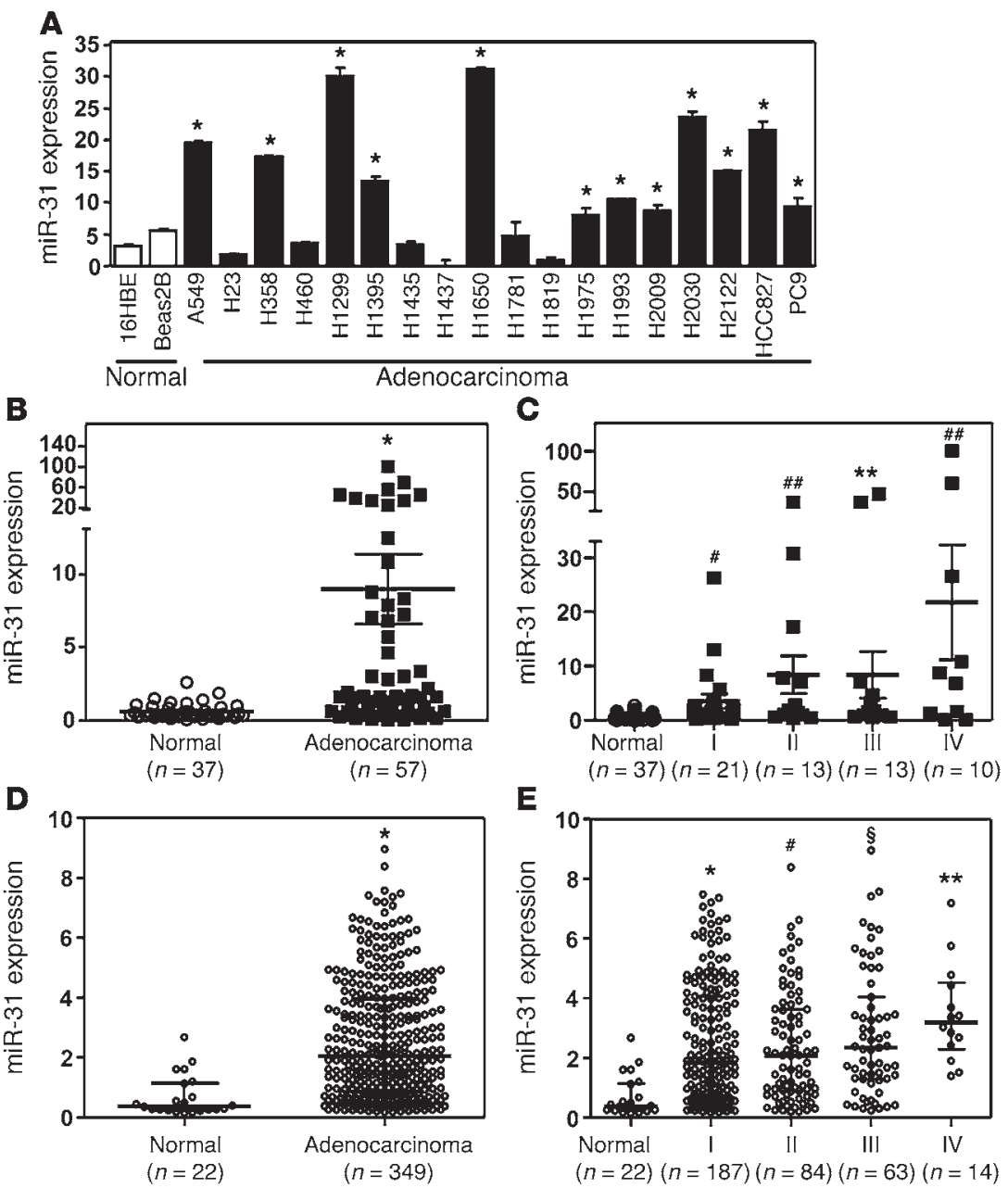

E

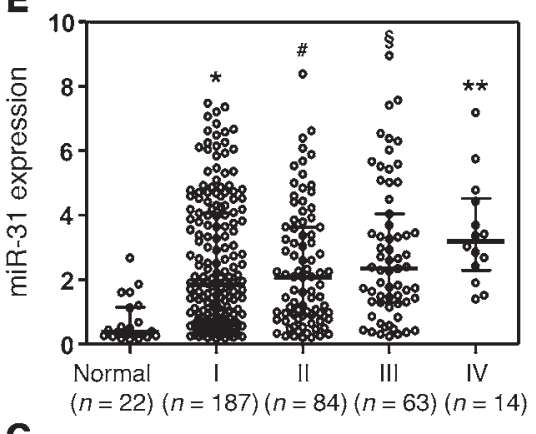

G
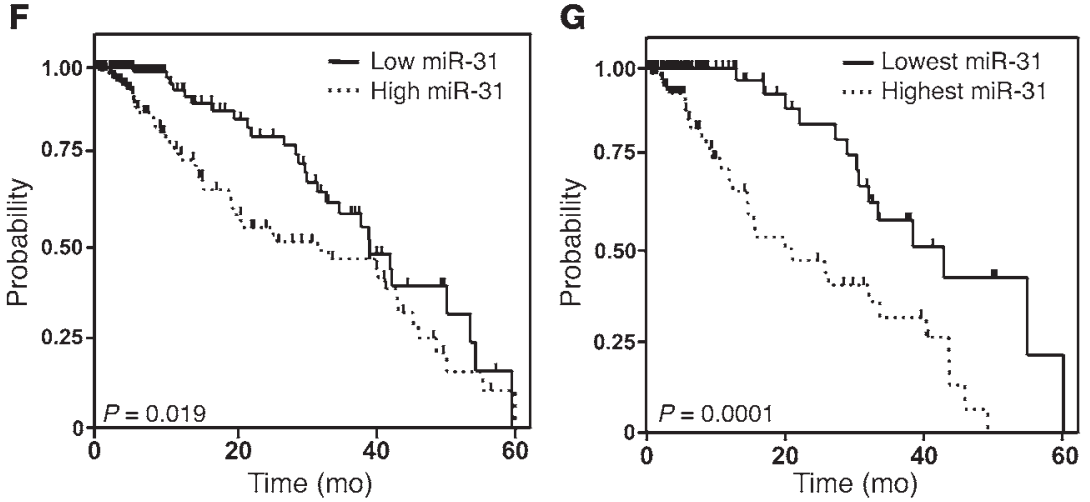

Figure 1. $\mathrm{miR}-31$ is overexpressed and correlates with poor survival in lung adenocarcinoma. (A) qRT-PCR analysis (performed in triplicate) of miR-31 expression in human lung epithelial and adenocarcinoma cells lines. $\Delta C \mathrm{C}$ values graphed are relative to the endogenous control RNU6B small RNA with SEM. ${ }^{*} P \leq 0.0012$ $t$ test. Data are representative of 3 independent experiments. (B and $\mathbf{C}$ ) qRT-PCR analysis of miR-31 expression in normal human lung tissue and (B) all human lung adenocarcinoma samples or (C) samples separated by stage of disease. miR-31 expression relative to RNU6B; mean values are indicated by solid bars, and values are shown as mean $\pm \mathrm{SEM}$. ${ }^{*} P=0.004$; ${ }^{\#} P=0.0009 ;{ }^{*} P=0.0017 ;{ }^{\#} P=0.0001, t$ test. The number of samples is shown. (D) miR-31 expression in normal lung and lung adenocarcinomas from TCCA miR expression profiles displayed as $\log _{2}$-transformed values normalized to reads per million. ${ }^{*} P=4.99 \times 10^{-14}$, $t$ test. (E) Data from $\mathbf{D}$ separated by stage. ${ }^{*} P=4.8 \times$ $10^{-13} ;{ }^{\#} P=5.3 \times 10^{-10} ;{ }^{\S} P=3.4 \times 10^{-10} ;{ }^{* *} P=9.3 \times 10^{-06}$, $t$ tests. The number of samples is shown. ( $\mathbf{F}$ and $\mathbf{G}$ ) Kaplan-Meier analysis with (F) median (158 samples for each) and (G) lowest $(n=79)$ and highest $(n=79)$ quartiles of miR-31 expression for lung adenocarcinoma from TCGA expression data. $P$ values were determined by log-rank test.

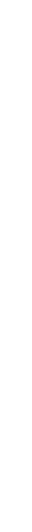

\section{Results}

miR-31 is overexpressed in human NSCLC. Given the limited understanding of the role of miR-31 in NSCLC, we examined miR-31 expression using quantitative real-time PCR (qRT-PCR) in a panel of human lung adenocarcinoma cells lines. We determined that approximately $70 \%$ (12 of 18) of the cell lines had significantly increased miR-31 expression compared with that of immortalized human lung epithelial cells (Figure 1A). To assess whether these data are reflected in patients, we examined miR-31 levels in frozen human lung adenocarcinoma and normal lung samples (clinicopathologic and demographic information in Supplemental Table 1; supplemental material available online with this article; doi:10.1172/JCI82720DS1). Lung adenocarcinoma had a 14-fold increase of mean miR-31 expression when compared with that of normal lung (Figure 1B). When miR-31 expression was analyzed by pathological stage (I-IV), it was significantly increased in each stage (Figure 1C). Moreover, there was a progressive elevation of miR-31 as pathological stage increased and lung adenocarcinomas became metastatic. Stage I lung adenocarcinomas expressed 5.7-fold more miR-31 than normal lung, and this rose to 13.5-fold in stages II and III and 35-fold in stage IV.

To further investigate miR-31 expression in lung adenocarcinoma, we evaluated NSCLC adenocarcinoma miR-sequencing data from The Cancer Genome Atlas (TCGA). As we observed in our patient cohort, miR-31 levels were significantly increased in lung adenocarcinoma (20-fold) compared with those in normal lung in the TCGA cohort (Figure 1D). miR-31 expression significantly increased 
Table 1. Analysis of 5-year overall survival in patients with lung adenocarcinoma from the TCGA data set

Variables

\begin{tabular}{ll} 
& HR \\
Age & 1.49 \\
\hline Tumor size & 3.18 \\
\hline Tumor stage $^{\mathrm{A}}$ & 3.55 \\
LN $^{\mathrm{B}}$ metastasis & 2.71 \\
miR-31 expression $^{\mathrm{C}}$ & 3.53
\end{tabular}

Univariate

$95 \% \mathrm{Cl}$

$0.79-2.80$

$1.28-7.87$

$1.89-6.65$

$1.46-5.04$

$1.79-6.95$

$\begin{array}{cc}\boldsymbol{P} \text { value } & \text { HR } \\ 0.22 & \\ 0.012 & 1.75 \\ 7.60 \mathrm{E}-05 & 1.90 \\ 1.57 \mathrm{E}-03 & 1.62 \\ 2.70 \mathrm{E}-04 & 3.09\end{array}$

HR
Multivariate

$95 \% \mathrm{Cl}$

$P$ value

$\begin{array}{ll}0.65-4.72 & 0.272\end{array}$

$0.80-4.53 \quad 0.148$

$0.71-3.69 \quad 0.253$

$1.51-6.32 \quad 1.93 \mathrm{E}-03$

${ }^{A}$ Early stages (I and II) versus late stages (III and IV); ${ }^{B} \mathrm{LN}$, Iymph node; ${ }^{C}$ top quartile versus bottom quartile.

as tumors progressed to disseminated disease (Figure 1E). Notably, miR-31 levels in stage IV lung adenocarcinoma were not only statistically increased compared with those in normal lung but also when compared with all other tumor stages (Supplemental Table 2).

High miR-31 expression correlates with poor survival of patients with lung adenocarcinoma. Given the higher levels of miR-31 during adenocarcinoma progression, we investigated whether miR-31 cor- related with patient survival. Separating patients into two groups using the median expression of miR-31 as the dividing line revealed that patients with higher miR-31 expression had significantly worse 5 -year overall survival compared with that of patients with lower miR-31 expression ( $P=0.019$, log-rank test; Figure $1 \mathrm{~F})$. The median survival decreased by 7.3 months (39.0 vs. 31.7 months) when miR31 levels were high (hazard ratio [HR]: 1.76; 95\% CI: 1.09-2.84,
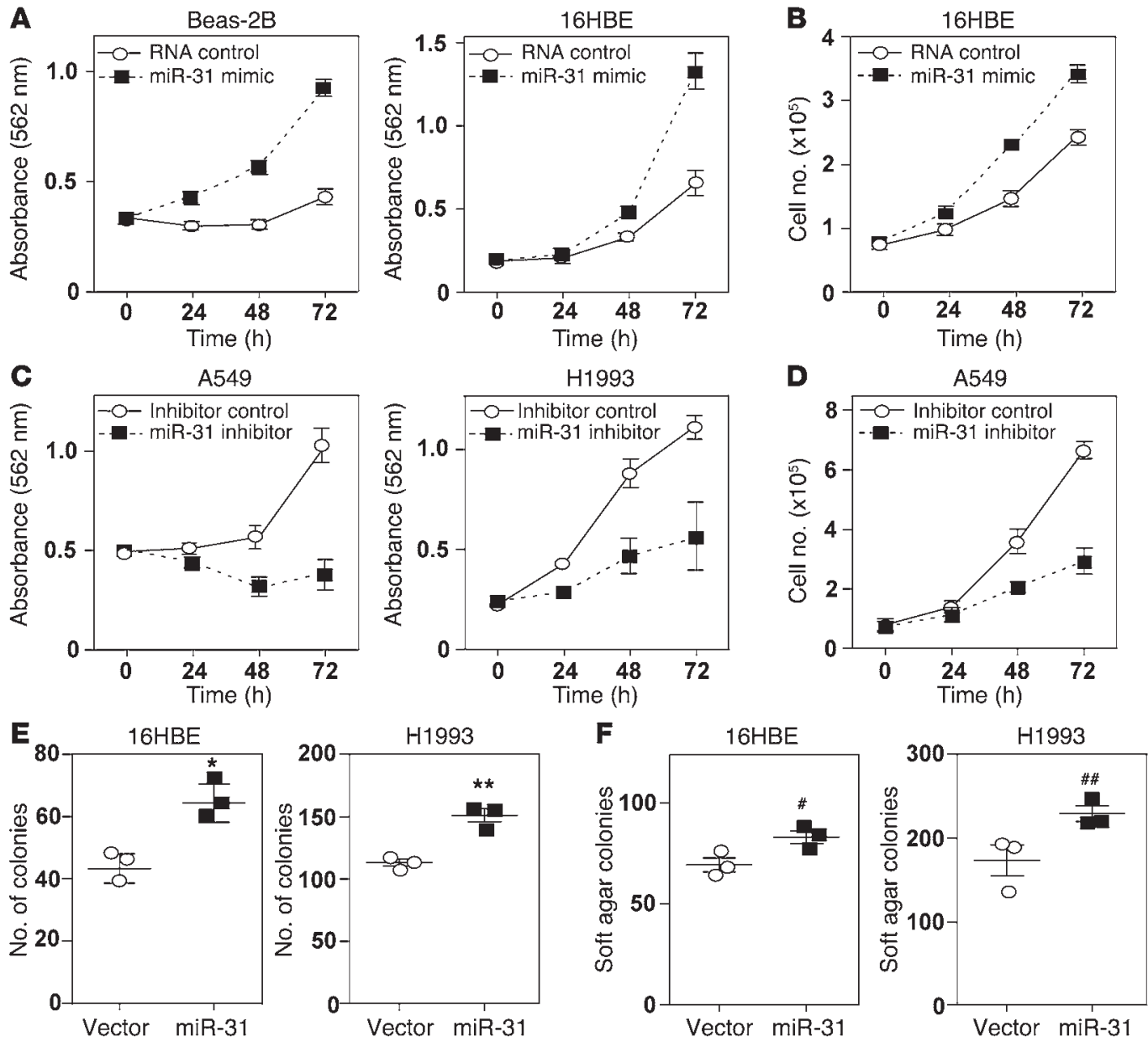

Figure 2. miR-31 promotes lung epithelial cell proliferation. (A and B) Beas-2B and 16HBE cells transfected with miR-31 mimic or RNA control (performed in triplicate). (A) MTT assays were performed, and (B) viable cells were counted at intervals. Data are representative of 3 independent experiments. (C and D) A549 and H1993 adenocarcinoma cells transfected with miR-31 inhibitor or inhibitor control (performed in triplicate). (C) MTT assays were performed, and (D) viable cells were counted at intervals. Data are representative of 3 independent experiments. (E and $\mathbf{F}$ ) The cells indicated were infected with a miR-31-encoded retrovirus or empty retrovirus (vector) control. (E) Clonogenic and (F) soft agar assays were performed in triplicate. Data are representative of 2 independent experiments. Colonies were counted after 14 days. ${ }^{*} P=0.0032 ;{ }^{*} P=0.0067 ;{ }^{*} P=0.023 ;{ }^{\#} P=0.027, t$ tests. 

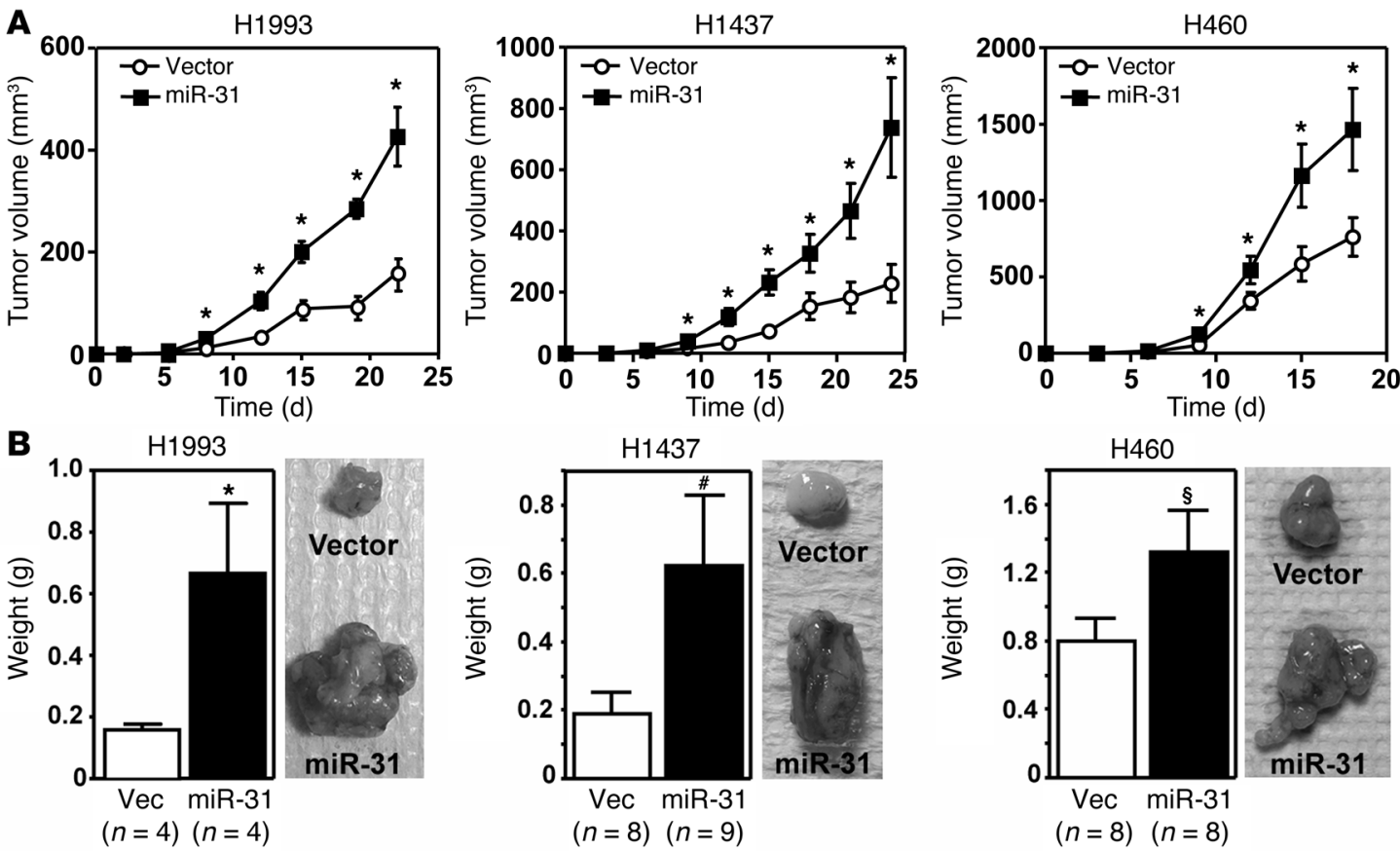

Figure 3. Elevated miR-31 increases adenocarcinoma cell growth in vivo. $\mathrm{H} 1993, \mathrm{H} 1437$, and $\mathrm{H} 460$ cells were infected with miR-31-encoded retrovirus (miR-31) or empty retrovirus (vector [vec]) control. Cells were injected subcutaneously into the flanks of nude mice, and (A) tumor volume was measured at intervals $\left({ }^{*} P<0.04, t\right.$ tests) and (B) tumor weight was measured $\left({ }^{*} P=0.034,{ }^{*} P=0.039\right.$, and ${ }^{\$} P=0.042$ respectively, $t$ tests) at time of sacrifice (day 22 , 24 , and 18, respectively). Tumor number indicated by $n$ values. Error bars indicate SEM. Representative pictures of tumors from $\mathbf{A}$ are shown in $\mathbf{B}$.

$P=0.02$, Wald test). There was an even greater overall decreased 5 -year survival for patients with the highest miR-31 (top quartile) expression compared with that of patients with the lowest (bottom quartile) expression $(P=0.0001, \log$-rank test; Figure $1 G)$, and they had a reduction of median survival of 21.4 months (42.2 vs. 20.8 months). Given the aggressiveness of NSCLC, 3-year survival was also assessed by dividing patients into two groups based on the median miR-31 expression. Patients with higher miR-31 expression levels had significantly worse 3-year overall survival compared with those with lower miR-31 expression levels $(P=0.00085$, logrank test; Supplemental Figure 1). The median survival decreased by 10.5 months (19.4 vs. 29.9 months) when miR-31 levels were high (HR: 2.61 ; 95\% CI: $1.45-4.68, P=0.001$, Wald test). Therefore, increased miR-31 expression significantly correlates with decreased survival of patients with lung adenocarcinoma.

To further investigate the effect of miR-31 expression on 5-year overall survival of patients with lung adenocarcinoma, univariate and multivariate Cox regression analyses were conducted. miR-31 expression and tumor size, tumor stage, and lymph node metastasis were significantly associated with overall survival in univariate analysis (Table 1). Considering these 4 variables, multivariate Cox regression analyses showed that miR-31 independently correlated with 5-year overall survival (Table 1). Similar results were obtained for multivariate analyses of 3-year overall survival (HR: 2.50; 95\% CI: $1.36-4.61, P=0.003$, Wald test). These results indicate that miR-31 is a predictive indicator and independent determinant of overall survival in patients with lung adenocarcinoma.

miR-31 promotes lung epithelial cell growth and xenograft tumor formation. To test the functional relevance of miR-31 overexpression in lung epithelial cells, we overexpressed or inhibited miR-31 and eval- uated the cellular outcomes. Increased miR-31 expression resulted in increased cell growth, as measured by MTT assay, in the immortalized, nonmalignant normal lung epithelial cell lines Beas-2B and 16HBE (Figure 2A). Cell counting determined that the differences detected by MTT assay were due to increased numbers of viable cells (Figure 2B and Supplemental Figure 2A). Overexpression of miR-31 in $\mathrm{H} 1437$ and $\mathrm{H} 460$ adenocarcinoma cells that expressed levels of miR-31 that are similar to those detected in the nonmalignant lung epithelial cells also resulted in increased cell growth and cell numbers (Supplemental Figure 2, B and C). Conversely, inhibition of miR-31 in the lung adenocarcinoma cell lines A549, which had high levels of miR-31, and H1993, which had moderately increased miR31 , resulted in decreased cell growth (Figure 2C), with reduced numbers of viable cells for both (Figure 2D and Supplemental Figure 2D). We did not observe any difference in the numbers of dead or dying cells with inhibition of miR-31 that could explain the decrease in cell number (Supplemental Figure 2E). However, evaluation of BrdU incorporation showed that miR-31 inhibition resulted in reduced numbers of BrdU-positive cells (Supplemental Figure 2F). Similarly, cell cycle analysis revealed a decrease in the number of cells in $\mathrm{S}$ phase and an increase in cells in $G_{1}$ following miR-31 inhibition (Supplemental Figure 2G), indicating that fewer adenocarcinoma cells were cycling when miR-31 was inhibited. Together, the data indicate that miR-31 is a proproliferative factor for lung epithelial cells and is necessary for the proliferation of adenocarcinoma cell lines in vitro.

To further investigate the effect of miR-31 on lung cell growth, we measured the ability of miR-31 to influence colony formation under conditions of low cell density. Both nonmalignant lung epithelial cells and adenocarcinoma cells were infected with a miR-31encoded retrovirus to obtain more than transient miR-31 overex- 
A

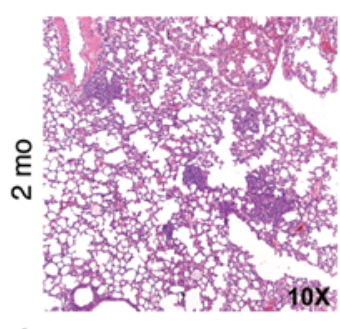

C

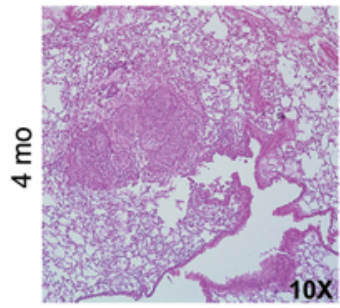

$\mathbf{F}$

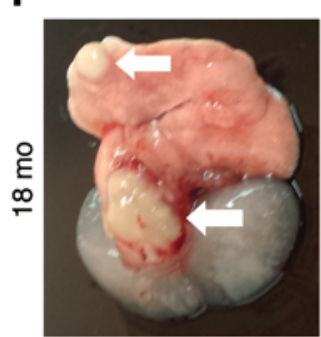

I

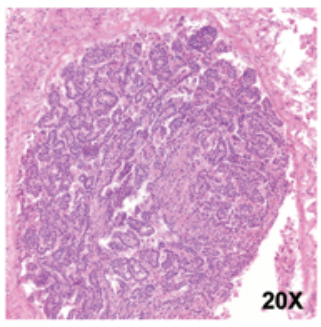

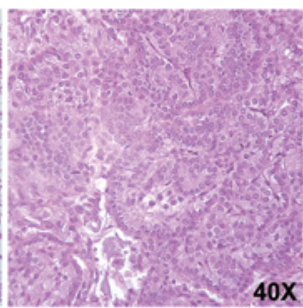

G
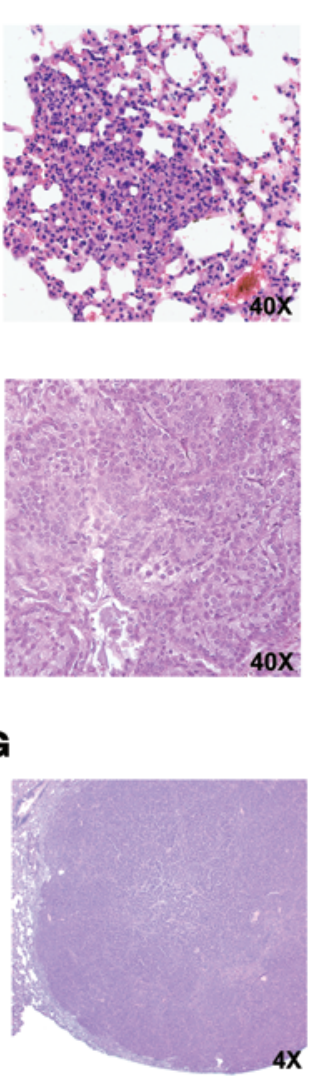

J

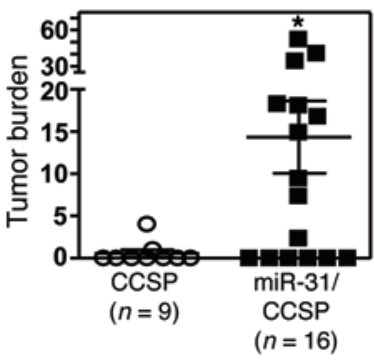

D

H
B
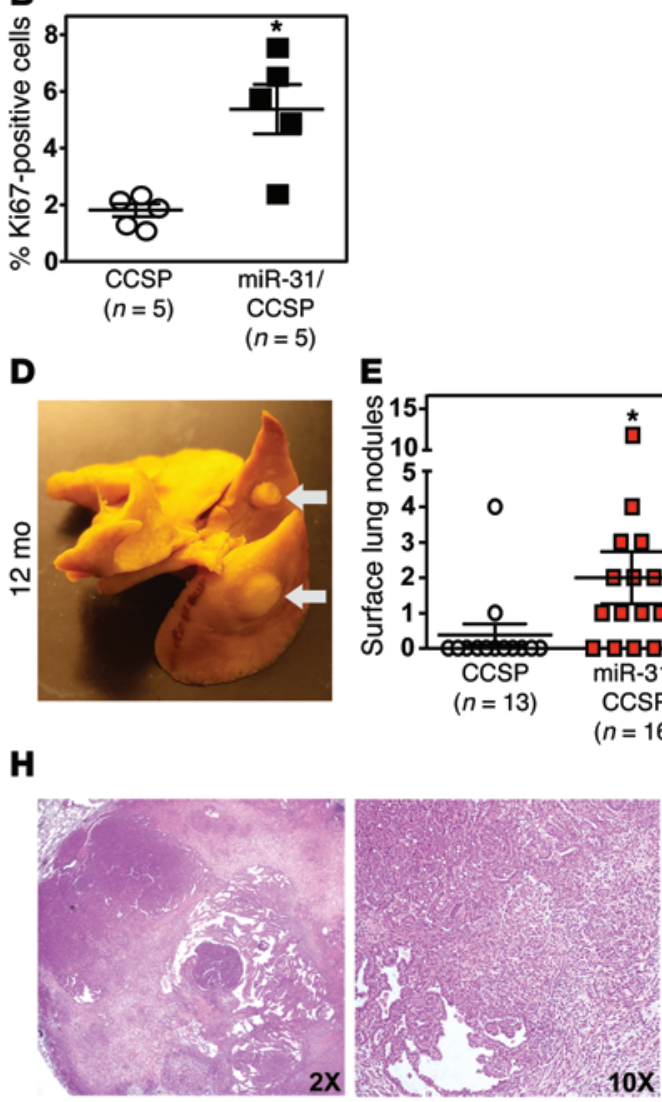

$\mathbf{K}$

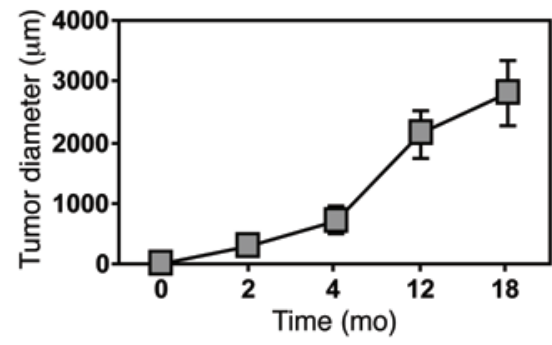

Figure 4. miR-31 initiates benign and malignant lung tumor formation in vivo. Cohorts of miR-31/CCSP and littermate CCSP control mice were given dox in their drinking water for (A and B) 2 months, (C) 4 months, (D and E) 12 months, or (F-J) 18 months. (A, C, and G-I) Representative photographs of H\&Estained lung sections from miR-31/CCSP mice (original magnification: $\times 2$ [H, left]; $\times 4$ [C] ; $\times 10[\mathbf{A}$ and $\mathbf{C}$, left, and $\mathbf{H}$, right]; $\times 20[\mathbf{I}] ; \times 40[\mathbf{A}$ and $\mathbf{C}$, right]). Larger histology pictures are shown in Supplemental Figure 5. (B) Ki67 IHC was performed on lung sections $\left({ }^{*} P=0.00124, t\right.$ test). The number of mice evaluated is denoted by $n$ values. ( $\mathbf{D}$ and $\mathbf{F})$ Representative photographs of (D) Bouin's fixed lungs and (F) gross lungs, with arrows pointing to tumors. (E) Lung surface tumor nodules were counted on Bouin's fixed lungs ( ${ }^{*} P>0.03, t$ test). The number of mice evaluated is denoted by $n$ values. (I) Representative photograph of an H\&E-stained section of lung adenocarcinoma showing invasion into a blood vessel. (J) Tumor burden was determined by comparing the area of tumor to that of normal lung from H\&E-stained lung sections $\left({ }^{*} P=0.0145, t\right.$ test). The number of mice evaluated is denoted by $n$ values. (K) The mean tumor diameter for lung tumors in miR-31/CCSP mice given dox for the indicated intervals was determined from H\&E-stained sections.

pression. All miR-31-overexpressing lines (nonmalignant and malignant) had significantly increased colony numbers compared with retroviral vector controls (Figure 2E and Supplemental Figure 2H). Elevated miR-31 levels also promoted soft agar colony growth in both nonmalignant and malignant lung epithelial cells (Figure 2F).

Next, we tested whether increased levels of miR-31 would promote lung adenocarcinoma growth in vivo with xenografts. H1993, H1437, and H460 lung adenocarcinoma cells that do not express high levels of miR-31 were infected with a retrovirus encoding miR-31 to overexpress miR-31 or an empty retrovirus control. Cells were injected into the flanks of athymic nude mice and monitored over time. For all 3 cell lines, the miR-31-overexpressing cells grew tumors at a faster rate compared with cells containing vector control (Figure 3A). At the time of sacrifice, miR-31-overexpressing H1993, H1437, and H460 tumors, which maintained elevated miR-31 levels (Supplemental Figure 2I), were significantly larger compared with vector controls (Figure 3A). In addition, miR-31-overexpressing tumors weighed 4,3 , and 1.5 times more, respectively, than tumors with vector control (Figure 3B). Therefore, miR-31 effectively promoted adenocarcinoma tumor growth in vivo. 
Table 2. Lung-specific miR-31 overexpression after 2 months

$\begin{array}{lcc}\text { Pathology } & \text { ACSP }(n=12) & \operatorname{miR}-31 / \operatorname{CCSP}(n=13) \\ \text { No lesions } & 12(100 \%) & 6(46.2 \%) \\ \text { Hyperplasia } & 0 & 7(53.8 \%) \\ \text { Adenoma } & 0 & 1(7.7 \%)\end{array}$

${ }^{A}$ Determined from $\mathrm{H} \& \mathrm{E}$-stained lung sections. Lungs may have both hyperplasia and adenoma and, thus, be counted in more than one row. $n$ is the number of mice analyzed.

Elevated miR-31 initiates lung hyperplasia and benign and malignant tumor growth in vivo. Our data indicate that miR-31 is proproliferative and tumor growth promoting in lung cells. To determine whether miR-31 could initiate lung tumorigenesis in vivo, we generated doxycycline-inducible (dox-inducible) miR-31 transgenic mice (tet-O-mmu-miR-31 mice; Supplemental Figure 3A). These miR-31 mice were crossed with CCSP-rtTA transgenic mice to generate mice that allowed us to overexpress miR-31 specifically in the lung. There was a typical Mendelian inheritance of the transgene (Supplemental Figure 3B). To verify that miR-31 could be induced in the lungs of this new mouse model, we provided wildtype and single- and double-transgenic mice either control water or dox-containing water for 2 weeks. Evaluation of miR-31 showed that dox induced miR-31 expression specifically in the lungs of the double-transgenic tet-O-mmu-miR-31/CCSP-rtTA mice (miR-31/ CCSP mice), whereas none of the controls showed changes in miR-31 expression (Supplemental Figure 3C). Moreover, miR-31 was expressed at elevated, but physiologically relevant, levels in the lungs of dox-exposed miR-31/CCSP mice.

We generated a cohort of single- and double-transgenic mice to investigate the effects of miR-31 on lung tumor development, and lungs from mice on dox were examined at intervals. After only 2 months of miR-31 induction (Supplemental Figure 4), we observed microscopic lesions in the lungs, with hyperplasia detected in half of the miR-31/CCSP mice and an adenoma present in 1 of the $13 \mathrm{miR}-31 / \mathrm{CCSP}$ mice evaluated (Figure 4A, Table 2, and Supplemental Figure 5A). Compared with lungs from littermate control CCSP mice, lungs from miR-31/CCSP mice had significantly more proliferating cells, as detected by Ki67 positivity (Figure 4B and Supplemental Figure 5B). After 4 months of miR-31 induction, the lung lesions were larger (Figure $4 \mathrm{C}$ and Supplemental Figure 5C), and over $60 \%$ of the miR-31/CCSP mice had lung hyperplasia and approximately $20 \%$ had an adenoma (Table 3 ). In contrast, none of the control littermates had lung hyperplasia or adenomas after 2 or 4 months of exposure to dox (Tables 2 and 3). Additionally, none of the $5 \mathrm{miR}-31 / \mathrm{CCSP}$ littermates that were administered control sugar water for 4 months developed lung lesions. Therefore, $62 \%$ and $82 \%$ of the miR-31-induced miR-31/ CCSP mice had hyperplasia and/or benign tumors of the lung by 2 and 4 months, respectively.

We next examined lungs after prolonged miR-31 induction (12 and 18 months). After 12 months of increased miR-31 expression (Supplemental Figure 4), we observed numerous large and small macroscopic surface lesions on the lungs of miR-31/CCSP mice (Figure 4D). Quantification of surface lung nodules determined that $70 \%$ of the miR-31/CCSP mice on dox had surface lung nodules. However, only $15 \%$ of the control CCSP littermates on dox had surface lung nodules (Figure 4E), which is the expected frequency in the FVB tumor-prone mice (23).

After 18 months of induced miR-31 expression, we observed an increase in the size of macroscopic lung nodules in miR-31/CCSP mice (Figure $4 \mathrm{~F}$ ). In addition, we determined microscopically that over $50 \%$ of miR-31/CCSP mice on dox had very large lung adenomas (Figure $4 \mathrm{G}$ and Supplemental Figure 5D), and many of these mice also had multiple adenomas (Table 4). We observed small adenomas in 2 control CCSP mice, which is the expected frequency in FVB mice (23). Notably, a quarter of miR-31/CCSP mice developed lung adenocarcinoma (Table 4, Figure $4 \mathrm{H}$, and Supplemental Figure 5D). The adenocarcinomas that developed were typically invasive, showing invasion into and blocking of blood vessels (Figure 4I and Supplemental Figure 5D). Additionally, the overall tumor burden in miR-31/CCSP mice was significantly greater than that in controls (Figure 4J). Measurement of the size of tumors that formed in the miR-31/CCSP mice at each interval evaluated showed that tumors increased in proportion to the amount of time miR-31 had been induced (Figure 4K). These data demonstrate that elevated miR-31 expression in the lung initially results in epithelial cell hyperproliferation and the formation of benign tumors and that prolonged miR-31 overexpression results in malignant lung tumor development.

miR-31 promotes mutant KRAS-driven tumorigenesis. Given that miR-31 was proproliferative and initiated lung hyperplasia and tumor development, we hypothesized that miR-31 would cooperate with an oncogenic driver of lung cancer. Because mutant KRAS is the most common NSCLC driver mutation in tumors from patients with lung adenocarcinoma $(7,8)$, we tested whether miR-31 could cooperate with mutant KRAS in lung tumorigenesis. We generated triple-transgenic tet-O-miR-31/CCSP-rtTA/ tet-O-KRas ${ }^{G 12 D}$ (miR-31/CCSP/KRAS ${ }^{\mathrm{G} 12 \mathrm{D}}$ ) mice and control double-transgenic CCSP-rtTA/KRas ${ }^{G 12 D}$ (CCSP/KRAS ${ }^{\mathrm{G} 12 \mathrm{D}}$ ) mice. Evaluation of lungs after 2 months of dox exposure showed that miR31 overexpression resulted in a significant increase in the number of proliferating lung cells (Ki67-positive cells) (Figure 5A). There was a small increase (15\%) in the number of surface lung nodules in miR-31/CCSP/KRAS ${ }^{\mathrm{G} 12 \mathrm{D}}$ mice compared with that in CCSP/ $\mathrm{KRAS}^{\mathrm{G} 12 \mathrm{D}}$ littermates (Figure $5 \mathrm{~B}$ ). Although the percentage of double- and triple-transgenic mice presenting with hyperplasia and adenoma was the same (Supplemental Figure 6A), the numbers of hyperplastic lesions (Figure 5C) and adenomas (Figure 5D) per section of lung were significantly increased in the miR-31/CCSP/

\section{Table 3. Lung-specific miR-31 overexpression after 4 months}

$\begin{array}{lcc}\text { Pathology } & \\ \text { N } & \operatorname{CCSP}(n=6) & \operatorname{miR}-31 / \operatorname{CCSP}(n=11) \\ \text { No lesions } & 6(100 \%) & 3(27.3 \%) \\ \text { Hyperplasia } & 0 & 7(63.6 \%) \\ \text { Adenoma } & 0 & 2(18.2 \%)\end{array}$

ADetermined from H\&E-stained lung sections. Lungs may have both hyperplasia and adenoma and, thus, be counted in more than one row. $n$ is the number of mice analyzed. 
Table 4. Lung-specific miR-31 overexpression after 18 months

\begin{tabular}{|c|c|c|}
\hline Pathology ${ }^{A}$ & $\operatorname{CCSP}(n=9)$ & $\mathrm{miR}-31 / \operatorname{CCSP}(n=16)$ \\
\hline No tumors & $7(77.8 \%)$ & $6(37.5 \%)$ \\
\hline Adenoma & $2(22.3 \%)$ & $9(56.3 \%)$ \\
\hline Multiple adenomas & 0 & $5(31.3 \%)$ \\
\hline Adenocarcinoma & 0 & $4(25.0 \%)$ \\
\hline
\end{tabular}

ADetermined from H\&E-stained lung sections. Lungs may have more than one tumor or tumor type and, thus, be counted in more than one row. $n$ is the number of mice analyzed.

KRAS ${ }^{\mathrm{G} 12 \mathrm{D}}$ mice. Additionally, the tumors in the miR-31/CCSP/ KRAS $^{\mathrm{G} 12 \mathrm{D}}$ mice were larger (Figure $5, \mathrm{E}$ and $\mathrm{F}$, and Supplemental Figure $6 \mathrm{~B}$ ) as was the overall tumor burden (Figure $5 \mathrm{G}$ ). During our analyses of lung sections, we consistently observed that miR31-overexpressing tumors were not as compact and exhibited patterns of infiltrative growth and severe dysplasia compared with the tumors in the double-transgenic controls (Supplemental Figure 6C). There was no difference in $K R a s^{G 12 D}$ expression between the miR-31/CCSP/KRAS ${ }^{\mathrm{G} 12 \mathrm{D}}$ and the CCSP/KRAS ${ }^{\mathrm{G} 12 \mathrm{D}}$ mice (Supplemental Figure 6D). However, due to the presence of the miR31 transgene, in miR-31/CCSP/KRAS ${ }^{\mathrm{G} 12 \mathrm{D}}$ mice administered doxcontaining water, there was increased miR-31 expression, which was at levels similar to those in miR-31/CCSP mice, compared with those in any of the littermate controls given dox-containing or control sugar water (Supplemental Figure 6E).

A separate cohort of miR-31/CCSP/KRAS ${ }^{\mathrm{G} 12 \mathrm{D}}$ and CCSP/ KRAS $^{\text {G12D }}$ mice was exposed to dox for 10 months to assess lung tumor development after prolonged miR-31 overexpression. Lungs from mice with elevated miR-31 expression showed increased tumor size (Figure 5H). Although there were no differences in the total number of atypical adenomatous hyperplasias or adenomas per section of lung between the two genotypes, the number of these lesions with anaplasia was significantly greater in lungs from miR-31/CCSP/KRAS ${ }^{\mathrm{G} 12 \mathrm{D}}$ mice (Figure 5, I and J). In addition, miR-31 overexpression resulted in an increased number of mice $\left(60 \%\right.$ of miR-31/CCSP/KRAS ${ }^{\mathrm{G} 12 \mathrm{D}}$ vs. $40 \%$ of CCSP/ KRAS $^{\text {G12D }}$ mice) with lung adenocarcinoma (Figure $5 \mathrm{~K}$ ). Additionally, $40 \%$ of the miR-31/CCSP/KRAS ${ }^{\mathrm{G} 12 \mathrm{D}}$ mice harbored multiple adenocarcinomas per lung, whereas none of the CCSP/KRAS ${ }^{\mathrm{G} 12 \mathrm{D}}$ mice that developed adenocarcinoma had more than one (Figure $5 \mathrm{~K}$ ). Therefore, miR-31 overexpression accelerated mutant KRAS-mediated tumor formation and increased the aggressiveness of the tumors that formed.

To further test the ability of miR-31 to promote oncogenic KRAS-driven lung tumors, we used a chemical carcinogenesis (urethane) model of lung cancer. A single dose of urethane gives rise to pulmonary lung carcinomas that develop in 15 to 20 weeks, of which the majority have activating KRAS mutations $(24,25)$. We injected miR-31/CCSP mice and single-transgenic CCSP control littermates with one dose of urethane and then administered dox to the mice for 6 weeks, the time at which adenomas, but not carcinomas, begin to form. miR-31 overexpression (Supplemental Figure 6F) increased the number of surface lung nodules (Figure $6 \mathrm{~A}$ ), lung tumor size (Figure 6B), and adenomas per lung section
(Figure 6C) compared with those in urethane-treated CCSP control mice. No adenocarcinomas were observed. Taken together, these results indicate that miR-31 cooperates with mutant KRAS to promote oncogenesis.

Given the cooperation between miR-31 and mutant KRAS we observed in lung tumorigenesis in mice, we sought to determine whether miR-31 overexpression was selected for in human lung adenocarcinomas with mutant KRAS. Evaluation of the TCGA data showed that miR-31 was similarly overexpressed in lung adenocarcinomas with mutant KRAS compared with tumors with wild-type KRAS (Supplemental Figure 6G). Therefore, increased levels of miR-31 are not exclusive to lung adenocarcinoma with mutant KRAS but instead appear to be an alteration that is common among lung adenocarcinomas.

miR-31 directly targets multiple negative regulators of RAS/ MAPK signaling. We used 3 prediction algorithms to identify potential mRNA targeted by miR-31 to gain insight into the mechanism for how miR-31 promotes tumor initiation and growth (Figure 7A). Those targets that overlapped between the 3 programs were sorted by gene ontology terms using the Database for Annotation, Visualization and Integrated Discovery $(26,27)$. Potential target genes were identified for miR processing, cell cycle, adhesion, and cell movement (Figure 7A). Notably, 4 genes (SPRED1, $S P R E D 2, S P R Y 1$, and RASA1) that negatively regulate RAS/ MAPK signaling were identified (Figure 7A). Because prediction algorithms are not likely to be able to identify all mRNA targets of a particular miR, we also aligned and evaluated the $3^{\prime}$-UTR sequences of each of the other family members of the negative regulators of RAS/MAPK signaling for potential miR-31-binding sequences. We determined that the 3'-UTRs of SPRY3 and SPRY4 had potential miR-31-binding regions as well. These in silico analyses were done for mice and humans and showed that miR31 had potential binding regions in these 6 putative target mRNA for both mouse and human $3^{\prime}$-UTR sequences.

To test whether the mRNA that had potential miR-31-binding regions were actual targets of miR-31, we performed a series of experiments. We first overexpressed miR-31 in nonmalignant lung epithelial cells and inhibited miR-31 in adenocarcinoma cell lines and analyzed the expression of 10 of the mRNA we identified as putative miR-31 targets. LATS2 has previously been described as a direct miR-31 target in lung cells (21), and our data were consistent with this finding (Figure 7, B and C). Additionally, SATB2 and DICER 1 are miR-31 targets in colorectal cancer and squamous cell carcinoma of the lung, respectively $(18,28)$, and we observed changes in these 2 mRNA with both overexpression and inhibition of miR-31 (Supplemental Figure 7, A and B, respectively). Similar results were also observed for DGCR8 (Supplemental Figure 7, A and B). Importantly, miR-31 overexpression significantly decreased mRNA expression of all 6 negative regulators of RAS/ MAPK signaling, whereas it did not affect the expression of $L K B 1$, a tumor suppressor in lung cancer that does not contain a miR-31 3'-UTR-binding region (Figure 7B). Inhibition of miR-31 in lung adenocarcinoma cells resulted in an increase in the mRNA of the 6 genes and no change in LKB1 (Figure 7C). We then focused on the 6 negative regulators of RAS/MAPK signaling and evaluated their protein levels following modulation of miR-31. When miR31 was overexpressed in lung epithelial cells, protein levels of all 
A

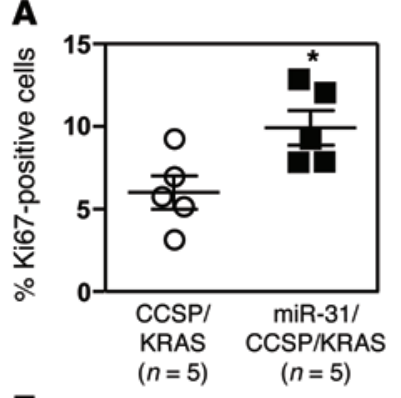

E

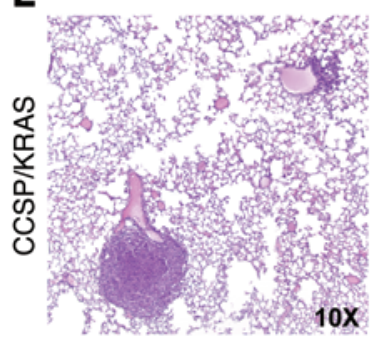

H

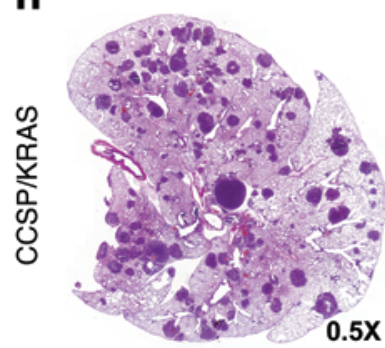

$\mathbf{J}$

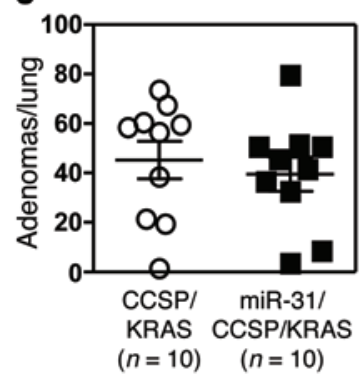

B
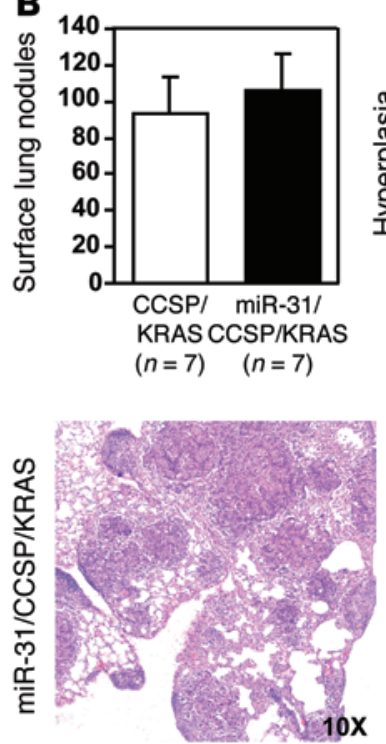

I
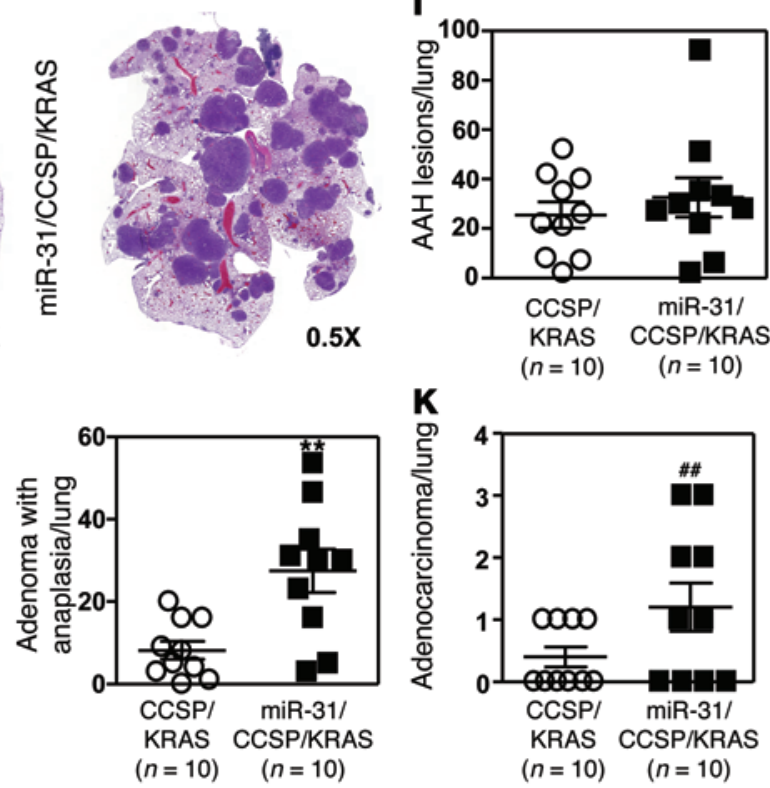

F

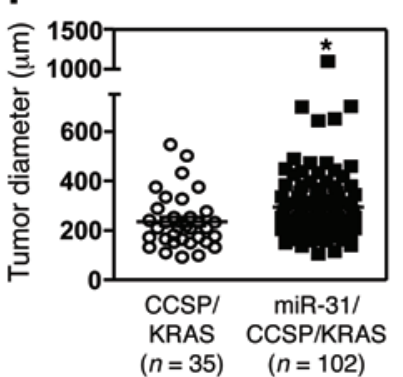

C

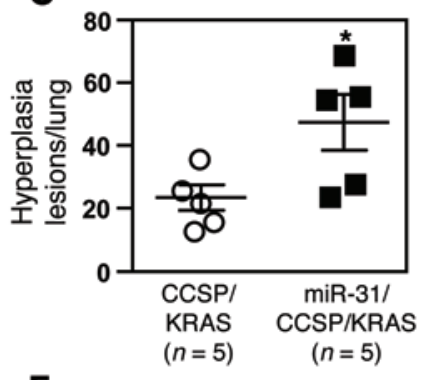

D

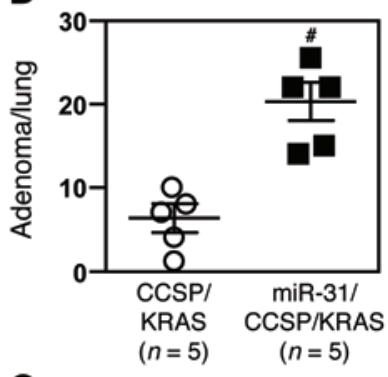

G

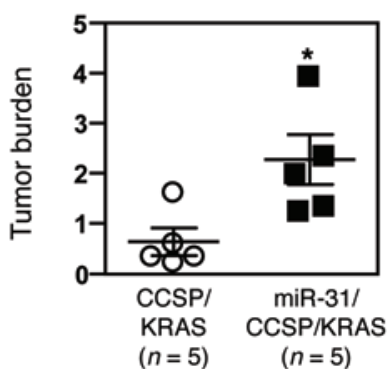

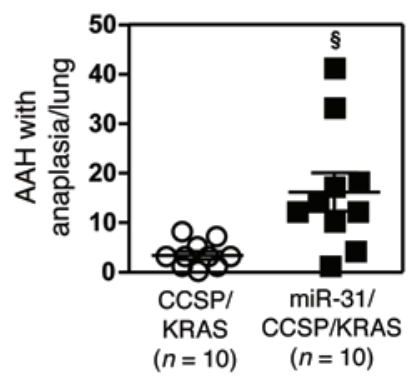

Figure 5. miR-31 promotes KRAS ${ }^{6120}$-driven lung tumorigenesis. Cohorts of miR-31/CCSP/KRAS ${ }^{\mathrm{C} 12 \mathrm{D}}$ and littermate CCSP/KRAS ${ }^{\mathrm{C} 12 \mathrm{D}}$ control mice were given dox in their drinking water for (A-G) 2 months or (H-K) 10 months. (A) Ki67 IHC was performed on lung sections $\left({ }^{*} P=0.00208, t\right.$ test). (B) Surface lung nodules were counted; error bars represent SEM. (C, D, and I-K) The number of hyperplasia lesions, adenomas, and carcinomas in each mouse was determined from H\&E-stained lung sections ( ${ }^{*} P=0.02 ;{ }^{\#} P=0.0006 ;{ }^{\$} P=0.0024 ;{ }^{* *} P=0.0016 ;{ }^{\#} P=0.037, t$ tests). AAH, atypical adenomatous hyperplasia. (E and $\mathbf{H})$ Representative $H \& E$-stained sections of lungs (original magnification: $\times 0.5[H] ; \times 10[E])$. Larger histology pictures are shown in Supplemental Figure 6B. (F) The mean tumor diameter was determined from $\mathrm{H} \& \mathrm{E}$-stained sections ( ${ }^{*} P=0.01, t$ test). (G) Tumor burden was determined by comparing the area of tumor to that of normal lung from $\mathrm{H} \& \mathrm{E}$-stained lung sections $\left({ }^{*} P=0.01, t\right.$ test).

6 negative regulators of the RAS/MAPK pathway were decreased (Figure 7D and Supplemental Figure 7C). Additionally, inhibition of miR-31 in lung adenocarcinoma cells resulted in increased protein levels for these 6 putative miR-31 targets (Figure 7D and Supplemental Figure 7C).

To determine whether the genes of the 6 negative regulators of RAS/MAPK signaling are direct miR-31 targets, 3'-UTR luciferase assays were performed. Luciferase activity was reduced by miR-31 in cells expressing the $3^{\prime}$-UTR for RASA1 and the first part of SPRED1, SPRED2, SPRY1, SPRY3, and SPRY4, whereas there was no reduction in luciferase with the second part of the $3^{\prime}$-UTR of SPRED1 that does not contain predicted miR-31-binding sites (Figure 7E). The well-characterized oncogenic miR-17-5p was used as a negative control, as, aside from that of RASA1, it was not predicted to bind the 3'-UTRs tested. Therefore, miR-31 directly targeted the 3'-UTRs of all 6 of the negative regulators of RAS/MAPK signaling.

To evaluate whether miR-31 targeting of these 6 negative regulators of RAS/MAPK signaling contributed to the proliferation we observed with miR-31 overexpression, we expressed the cDNA of each target with miR-31 in H1993 cells. The increased prolifer- 

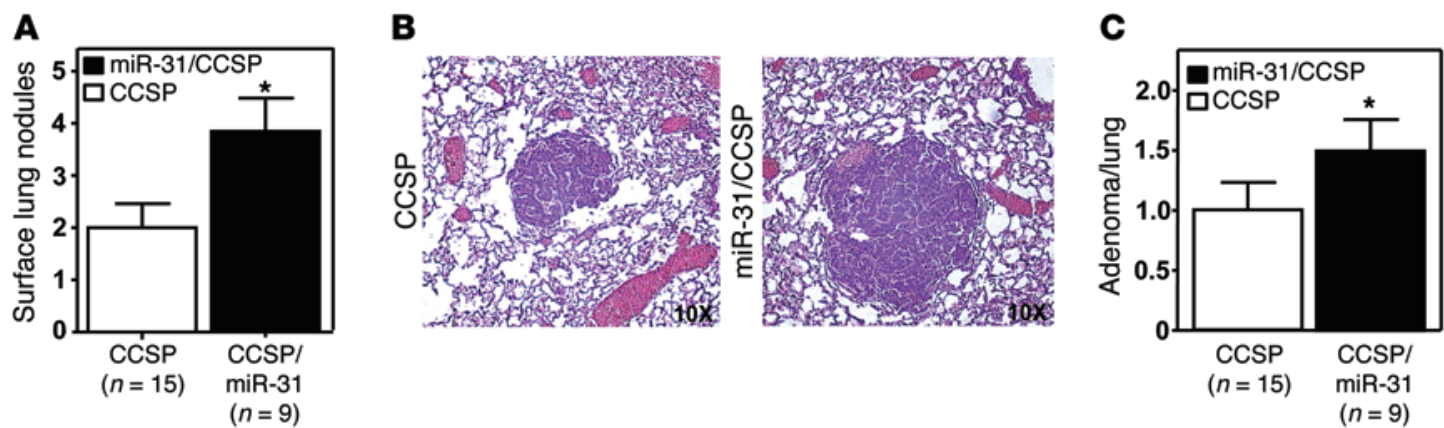

Figure 6. miR-31 enhances urethane-induced lung tumor growth. A cohort of miR-31/CCSP and littermate CCSP control mice was injected with urethane once and given dox for 6 weeks. (A) Surface lung nodules were counted; error bars represent SEM. ${ }^{*} P=0.03, t$ test. (B) Representative H\&E-stained sections (original magnification: $\times 10$ ). (C) The number of adenomas per mouse was determined from H\&E-stained sections. ${ }^{*} P=0.047, t$ test. The number of mice evaluated is denoted by $n$ values.

ation in the miR-31-overexpressing cells was completely inhibited with expression of RASA1, SPRED2, SPRY1, or SPRY4, whereas SPRED1 expression partially inhibited proliferation (Figure 7F). SPRY3 expression had no effect on proliferation. Taken together, the data indicate that the expression of 5 of the 6 negative regulators of RAS/MAPK signaling directly targeted by miR-31 can rescue the proproliferative effects of miR-31, restoring lung epithelial proliferation to normal levels.

Given that the 6 miR-31 targets have been described as negative regulators of MAPK signaling $(29,30)$, we examined the downstream consequence of MAPK signaling, ERK1/2 phosphorylation, when miR-31 levels were increased. Beas-2B lung epithelial cells transfected with miR-31 mimic showed increasing ERK1/2 phosphorylation with increasing miR-31 concentration by Western blot (Figure 8A). Moreover, with elevated levels of miR31 , there were higher basal levels of phosphorylated ERK1/2, as quantified with intracellular phospho-flow cytometry (Figure 8B). In addition, miR-31 overexpression allowed the cells to respond more rapidly and robustly to stimulation with serum, leading to an increased amplitude and duration of phosphorylated ERK1/2 (Figure 8B). To assess whether this increased RAS/MAPK signaling contributed to the proliferation induced by miR-31, we pharmacologically inhibited MEK. Specifically, 16HBE and Beas-2B cells transfected with either miR-31 mimic or RNA control were treated with two different MEK inhibitors (trametinib and selumetinib). Both MEK inhibitors partially blocked miR-31-induced proliferation (Figure 8C and Supplemental Figure 7D). Together, the data indicate that changes in miR-31 alter the protein levels of multiple negative regulators of RAS/MAPK signaling and elevated miR-31 results in increased ERK activation, leading to increased lung cell proliferation.

Reduced expression of the miR-31 targets, negative regulators of RAS/MAPK signaling, in human and mouse lung cancers. To determine whether the negative regulators of RAS/MAPK signaling that were direct targets of miR-31 had altered expression in human and mouse lung tumors, we first evaluated mRNA from a subset of the patients analyzed in Figure 1B. There was a significant decrease in RASA1, SPRED1, SPRED2, and SPRY4 mRNA in lung adenocarcinomas compared with mRNA in normal lung (Figure 9A). We observed a trend toward decreased SPRY1 and no decrease in SPRY3 mRNA. When expression was evaluated by tumor stage, SPRED1, SPRED2, and SPRY4 were significantly decreased at all stages, whereas RASA1 and $S P R Y 3$ were only significantly reduced at stage IV and stage I, respectively (Figure 9A). Regression analysis of these samples determined that increased miR-31 expression significantly correlated with decreased SPRED1 and SPRY4 mRNA expression and trended for SPRED2 and SPRY1 (Spearman correlation, Figure 9B). In addition, analysis of TCGA lung adenocarcinoma data revealed significant decreases in SPRED1, SPRY1, and SPRY4 compared with those in normal lung (Figure 9C), with trends toward decreased patient survival with reduced SPRED2 and SPRY1 (Supplemental Figure 8A). Additionally, there were significant inverse correlations between miR-31 expression and SPRED1, SPRED2, SPRY1, and SPRY4 levels (Supplemental Table 3).

We also analyzed mRNA expression in the lungs of miR-31/ CCSP mice exposed to dox for 2 months and 12 months and in the H1993 xenograft tumors from mice analyzed in Figure 3A. Three of the miR-31 target mRNA (Rasa1, Spred1, and Spry4) were decreased in the lungs of mice receiving dox for 2 months (Figure 10A). There were reduced levels of 4 of the miR-31 target mRNA (Rasa1, Spred1, Spred2, and Spry4) in mice in which miR-31 had been overexpressed for 12 months (Figure 10B). Five of the miR31 target mRNA (RASA1, SPRED1, SPRED2, SPRY1, and SPRY4) were downregulated in the human lung adenocarcinoma H1993 xenografts (Figure 10C), and all were decreased in the cultured H1993 adenocarcinoma cells used for the xenografts (Supplemental Figure 8B). SPRY3 mRNA was oddly increased in all the tissue samples evaluated (Figure 10, A-C). Western blot analysis of the targets in H1993 xenograft tumors showed decreases in 4 of the proteins (RASA1, SPRED1, SPRED2, and SPRY4) in tumors overexpressing miR-31 (Figure 10D). Taken together, the data demonstrate that miR-31 directly targets multiple negative regulators of RAS/MAPK signaling in murine and human lung cancers, which leads to increased MAPK signaling and tumorigenesis.

\section{Discussion}

Decreased miR-31 expression has been linked to increased tumorigenesis and metastasis in multiple malignancies (9-11, 13-16). However, miR-31 was recently shown to be upregulated in lymph node metastases of patients with lung adenocarcinoma and in lung tumors of cyclin E transgenic mice $(21,22)$. These conflicting 
A

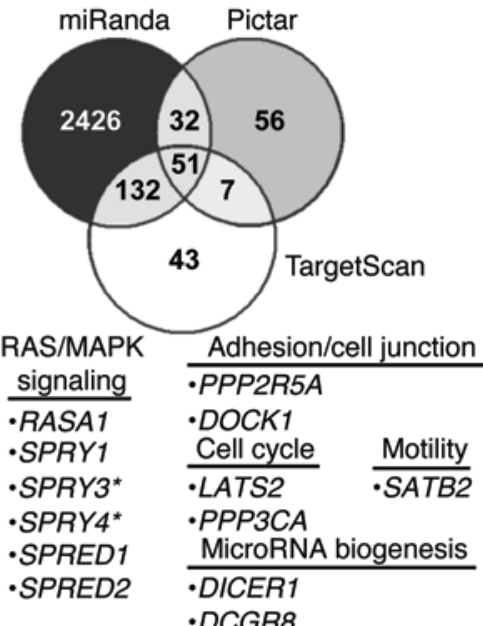

B

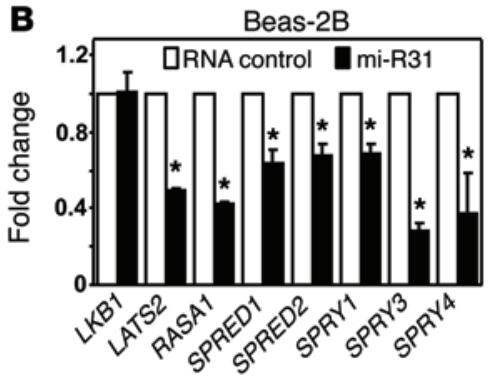

E

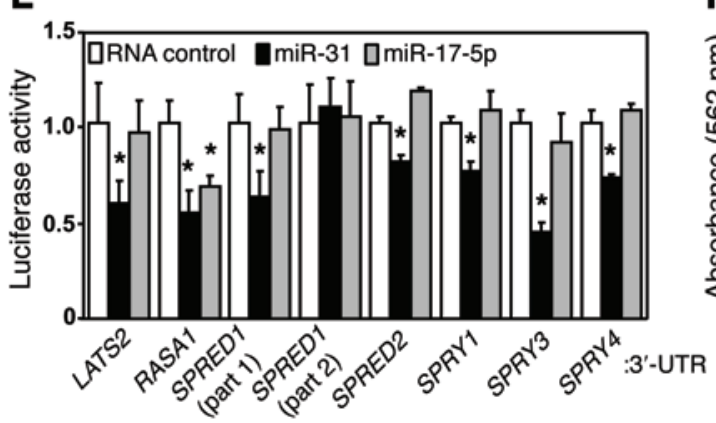

C

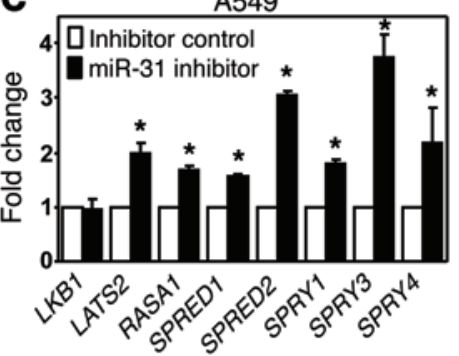

$\mathbf{F}$

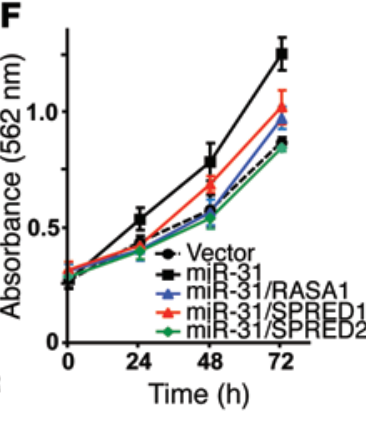

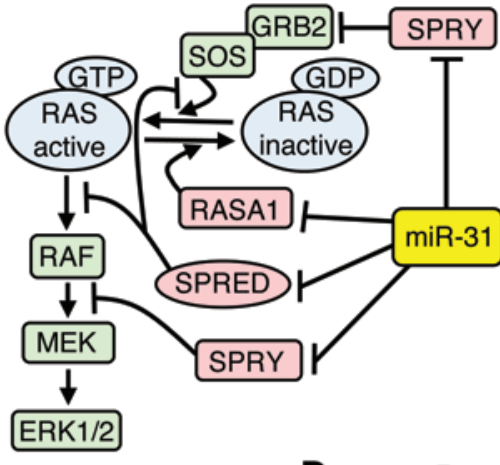
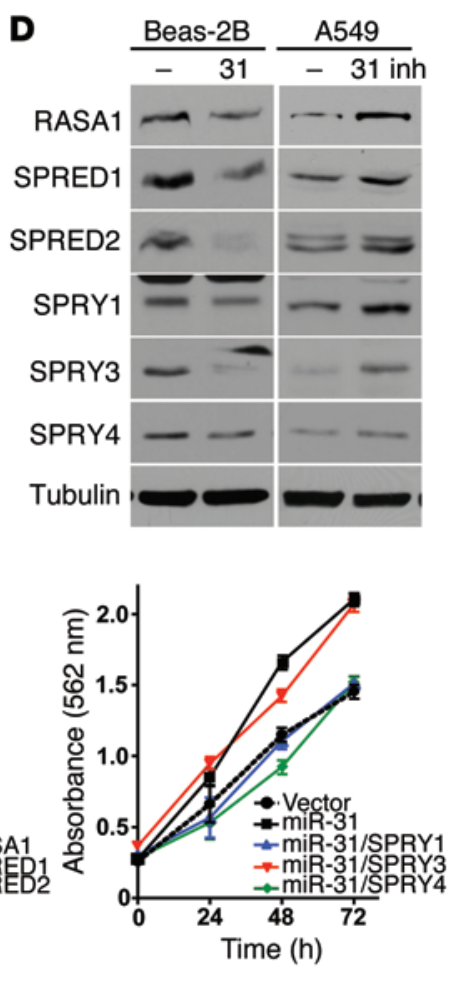

Figure 7. miR-31 directly targets multiple negative regulators of the RAS/MAPK pathway. (A) Shared putative miR-31 target mRNA identified from 3 prediction algorithms are listed. Asterisks denote mRNA that were manually aligned and determined to have a putative miR-31-binding region. Schematic of the RAS/MAPK signaling pathway. (B and C) qRT-PCR (performed in triplicate) for putative miR-31 target mRNA of (B) Beas-2B cells transfected with miR-31 mimic or RNA control or (C) A549 cells transfected with miR-31 inhibitor or inhibitor control. Values are normalized to $\beta$-actin levels. ${ }^{*} P<0.04, t$ tests. Data are representative of 3 independent experiments. (D) Beas-2B cells infected with miR-31-encoded (31) lentivirus or an empty lentivirus (-) and A549 cells transfected with miR-31 inhibitor (31 inh) or inhibitor control (-) were Western blotted. Densitometry results are shown in Supplemental Figure 7C. The data presented are representative of at least 2 independent experiments, and the data for both Beas-2B and A549 cells were from 3 separate experiments run on different gels. (E) 293T cells were transfected with luciferase vectors encoding all or a region of the 3'-UTR from each gene indicated (two parts of SPRED1 $3^{\prime}$-UTR) and miR-31 mimic, miR-17-5p mimic, or RNA control. Luciferase assays were performed (performed in triplicate); expression is relative to $\beta$-galactosidase activity. ${ }^{*} P<0.03, t$ tests. Data are representative of 4 independent experiments. (F) MTT assays of H1993 cells infected with a miR-31-encoded retrovirus or empty retrovirus (vector) and transfected with vectors encoding the indicated miR-31 target cDNA. Experiments are representatives of 2 independent experiments. Error bars represent SEM.

data for miR-31 highlight the tissue specificity or context dependence of some miR and indicate that the role of miR-31 in lung adenocarcinoma required further investigation. Our data significantly expand knowledge of miR-31 function in lung adenocarcinoma by revealing that miR-31 fits the classic definition of an oncogene in the lung and that it regulates RAS/MAPK signaling in lung cells, providing critical insight into its contribution to lung tumorigenesis. We show that miR-31 is not just overexpressed in late-stage metastatic disease but that it is overexpressed early in lung adenocarcinoma (stage I). Expression of miR-31 increased with tumor progression, with the highest levels in stage IV tumors. Our results showed that miR-31 has a proproliferative function in both nonmalignant and malignant lung epithelial cells and can drive tumor formation and carcinoma development in vivo in the lungs of mice; this provides an explanation for why its expression is selected for during lung tumorigenesis. Moreover, we demonstrated that miR-31 controls RAS/MAPK signaling by targeting multiple negative regulators of this pathway, providing a mecha- 
A
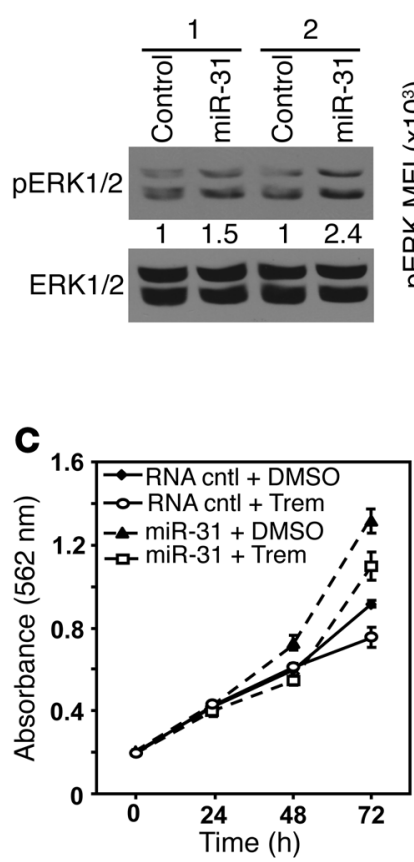

B
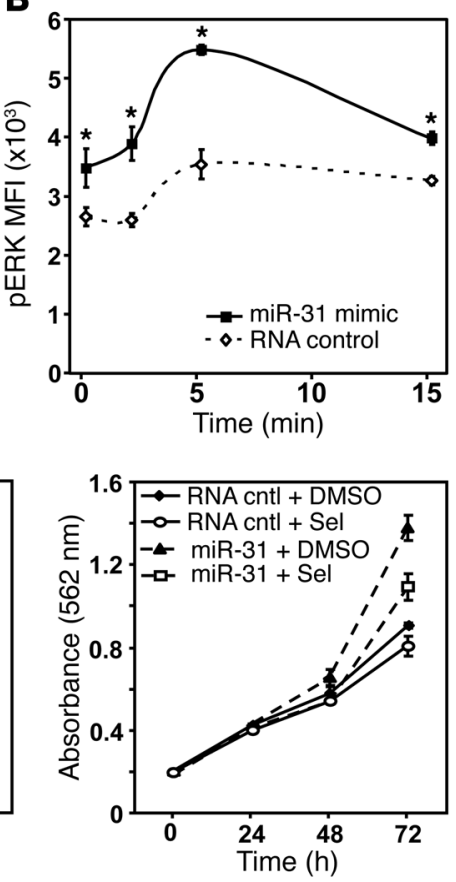

nism of its proliferation and tumor-inducing function in lung epithelial cells. Our data also revealed that miR-31 cooperated with mutant KRAS to accelerate lung tumorigenesis in vivo, highlighting a significant role for miR-31 in lung cancer.

We determined that miR-31 was significantly overexpressed in stage I lung adenocarcinoma. This increase of miR-31 levels during early tumor development and the proproliferative function of miR-31 led us to hypothesize that miR-31 alone may be able to initiate lung tumor development. Experiments showing that miR31 increases proliferation of lung adenocarcinoma cells and that elevated levels of miR-31 in mouse and human lung adenocarcinomas led other investigators to postulate that miR-31 was oncogenic in the lung $(21,22)$. We obtained similar data. However, here we also provide evidence that miR-31 is functioning as an oncogene in the lung in normal and nonmalignant lung cells. Our in vitro data show a proproliferative and protumorigenic function for miR-31 in nonmalignant lung epithelial cells. More importantly, we tested whether miR-31 can induce tumor growth in vivo specifically in the lung. We generated a transgenic mouse model that revealed miR31 overexpression in the lung-induced hyperplasia and small adenomas early on and formation of large adenomas and, in one-quarter of the mice, adenocarcinoma after prolonged overexpression. These in vitro and in vivo data with normal and nonmalignant lung epithelial cells indicate that miR-31 is an oncogene, and not just proproliferative, by eliciting the changes necessary for lung tumor development in vivo and inducing overt malignancy in the lung. These data show that miR-31 appears to be a better oncogene than mutant BRAF. Mutant BRAF activates MAPK/ERK signaling and is considered to be a driver mutation in numerous human malignancies, including those in lung (31); yet, mutant BRAF expression in the lungs of mice resulted in the growth of benign adenomas, which rarely progressed to carcinoma (32). Moreover, our finding that miR-31 only induced large adenoma and adenocarcinoma after prolonged overexpression was not entirely unexpected, since
Figure 8. RAS/MAPK signaling is altered by miR-31 and essential for miR-31-regulated lung cell growth. (A) Western blots for phospho-ERK1/2 (pERK1/2) and total ERK1/2 in Beas-2B cells transfected with miR-31 mimic or RNA control at two concentrations (200 and $400 \mathrm{nM}$, referred to in the figure as 1 and 2, respectively). Densitometry values are indicated. Data are representative of 2 independent experiments. (B) Beas-2B cells transfected (performed in triplicate) with miR-31 mimic or RNA control were evaluated by intracellular phospho-flow cytometry for phospho-ERK1/2 and total ERK1/2. The MFI of pERK is relative to total ERK $\left({ }^{*} P \leq 0.04, t\right.$ tests). Data are representative of 3 independent experiments. (C) $16 \mathrm{HBE}$ cells were transfected with miR-31 mimic or RNA control and treated with $100 \mathrm{nM}$ trametinib (Trem), selumetinib (Sel), or vehicle (DMSO) control. MTT assays were performed (performed in triplicate). Data are representative of 2 independent experiments.

miR modulate pathways and are not necessarily as powerful as the drivers of pathways, like the MYC and mutant RAS oncogenes. Importantly, our data show that miR-31 expression alone is sufficient to induce lung hyperplasia, precancerous tumors, and carcinoma in vivo, providing critical evidence that a miR can drive development of carcinoma, an epithelial-based cancer, in the lung.

Activating mutations in RAS are the leading driving mutations in lung adenocarcinoma, with the majority of those mutations occurring in KRAS (7). Combining miR-31 overexpression with mutant KRAS significantly accelerated malignant lung tumor development. Moreover, exposing mice that overexpressed miR31 in their lungs to the chemical carcinogen, urethane, which induces mutations in KRAS (24), also resulted in accelerated tumor development. These data illustrate that miR-31 cooperates with this oncogenic driver, facilitating lung epithelial cell transformation. Notably, the tumors that developed in miR-31/CCSP/ KRAS $^{\mathrm{G} 12 \mathrm{D}}$ mice had a growth pattern that was infiltrative at the edge of hyperplasic and occult tumor margins. However, we did not observe distant metastasis. This KRAS mouse model is known for not producing distant metastases, despite developing a large lung tumor burden with high-grade carcinoma (33). Consequently, we cannot exclude the possibility that miR-31 also contributes to lung cancer metastasis, as elevated levels of miR-31 were associated with patients with lung adenocarcinoma with lymph node metastasis, and this correlated with decreased survival (22). Increased miR-31 levels are also associated with advanced colorectal cancer and lung squamous cell carcinoma and correlated with decreased survival of patients with lung squamous cell carcinoma $(18,19)$. Moreover, our data suggest that SATB2 may be a target of miR-31 in lung cells, and downregulation of SATB2 has been associated with colorectal metastasis (34). Therefore, further studies on the role of miR-31 specifically in lung cancer metastasis will be needed to determine its role in this process and the targets involved.

The target mRNA reported for miR-31 in cancer types in which miR-31 inhibits tumorigenesis and suppresses metastasis are different than the target mRNA we identified in cancer types in which miR-31 is functioning in a proproliferative and protumorigenic capacity $(12,35,36)$. Additionally, given that an individual miR is predicted to bind hundreds of potential target mRNA, the notion that only one or two target mRNA are responsible for the large biological effects of dysregulation of a miR is unlikely $(2,37)$. It has been speculated that modest changes in several members of the same pathway lead to significant biological consequences $(2,38)$. 

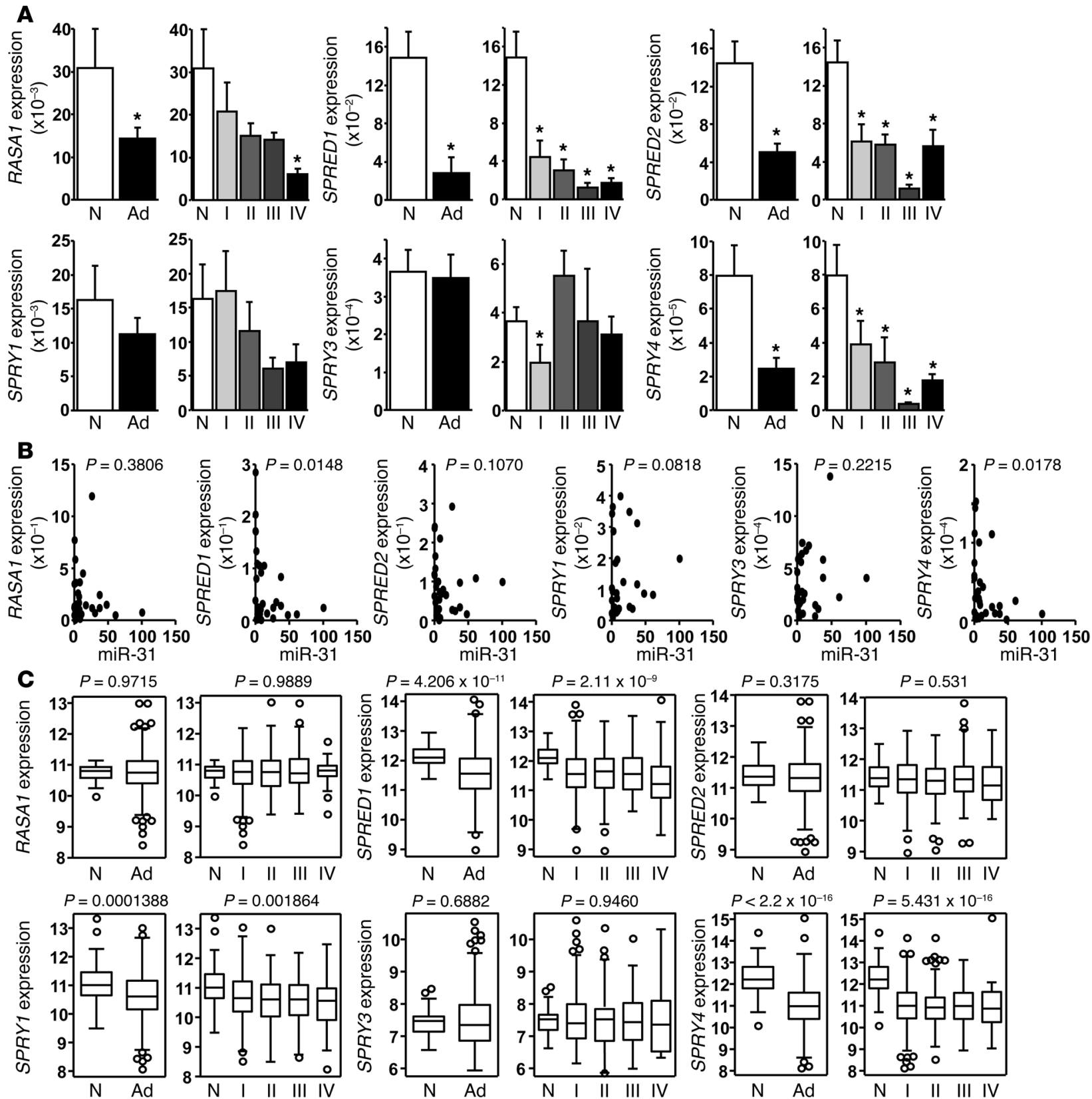

Figure 9. Reduced expression of negative regulators of the RAS/MAPK pathway with miR-31 overexpression in human lung adenocarcinoma. (A) qRTPCR analysis of the indicated miR-31 target mRNA in normal (N) human lung tissue and lung adenocarcinoma (Ad) (normal, $n=8 ;$ stage I, $n=7 ;$ stage II, $n=6$; stage III, $n=4$; stage IV, $n=6$ ). Values were normalized to $\beta$-actin levels. ${ }^{*} P<0.05, t$ tests. (B) Regression analysis for miR-31 and the indicated target mRNA in human lung tissue evaluated in A. $P$ values were determined by Spearman's rank correlation. (C) Expression of the 6 miR-31 targets in the lung adenocarcinoma TCGA RNA sequencing data set. Data are presented as $\log _{2}$ transformation of gene expression. $P$ values were calculated by Wilcoxon rank-sum test for normal versus tumor and Kruskal-Wallis rank-sum test for normal versus stages I-IV (normal $n=58$; stage I $n=279$; stage II $n=124$; stage III $n=84$; stage IV $n=27$ ). In box-and-whisker plots, horizontal bars indicate the mean values, boxes indicate 25 th to 75 th percentiles, and whiskers indicate 10th and 90th percentiles. Data represented by bar graphs in $\mathbf{A}$ are mean values. Error bars represent SEM.

In support of this concept, we identified 6 mRNA as direct targets of miR-31 in lung adenocarcinoma, and all are negative regulators of RAS/MAPK signaling (Figure 7A). RASA1 inhibits RAS-induced transformation by functioning as a negative regulator of RAS by promoting the inactive GDP-bound form of RAS (29). It was previously reported that miR-31 targets RASA1 in intrahepatic cholangiocarcinoma and colorectal cancer $(39,40)$, but whether miR-31 targeted it in the lung was unknown. The SPRED and SPRY genes are also suppressors of RAS signaling (30). The SPRY family member, SPRY2, which does not have a miR-31-binding site, was demonstrated, in vivo, to suppress KRAS-driven lung tumorigenesis (41), and SPRY4 expression is decreased in NSCLC cell lines (42). It has been postulated that the other SPRY genes have a similar function, but their roles in lung cancer are not entirely clear (43). We provide multiple lines of evidence that miR-31 directly targets mRNA of the 6 negative regulators of RAS/MAPK signaling and that these 

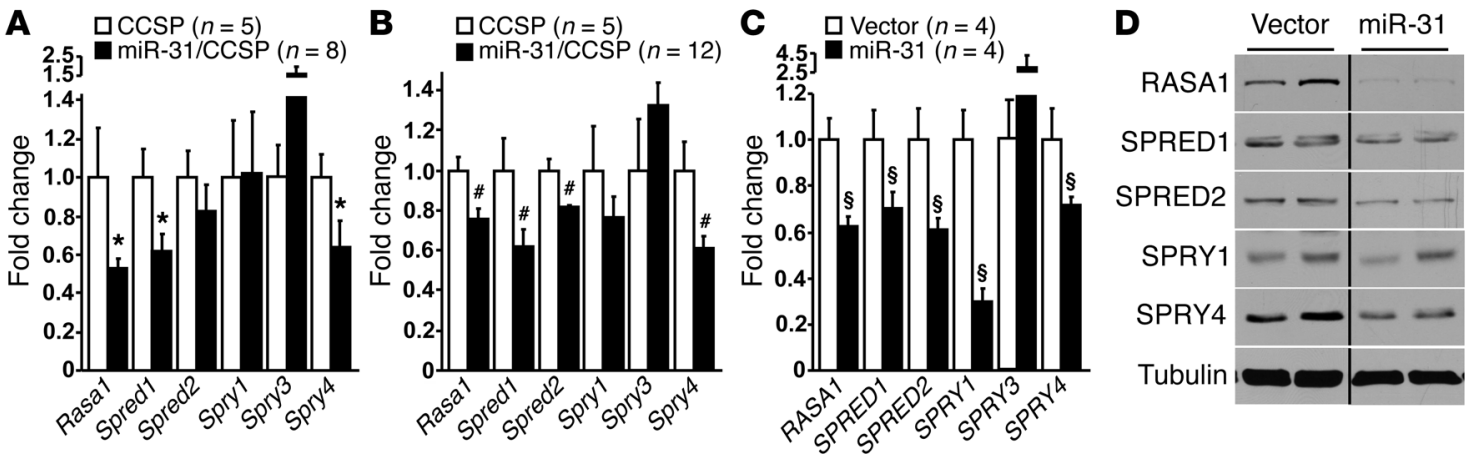

Figure 10. Decreased levels of negative regulators of the RAS/MAPK pathway in vivo with miR-31 overexpression. (A and B) qRT-PCR analysis of the indicated mRNA from the lungs of miR-31/CCSP and littermate CCSP transgenic mice that received dox for (A) 2 months or (B) 12 months from Figure 4 . Values are normalized to $\beta$-actin levels. The number of mice evaluated is denoted by $n$ values. (C) qRT-PCR for the indicated mRNA and (D) Western blot analysis of 2 tumors from each group of $\mathrm{H} 1993$ xenograft tumors from mice in Figure 3. Samples are from the same gel and exposure; noncontiguous samples are indicated by a black line. The number of tumors evaluated is denoted by $n$ values. Data represented by bar graphs in $\mathbf{A}-\mathbf{C}$ are mean values. Error bars represent SEM. ${ }^{*} P<0.05 ;{ }^{\sharp} P<0.05 ;{ }^{\circledR} P<0.05, t$ tests.

changes take place in human and mouse lung cancers. Notably, we observed reduced expression of at least 3 and as many as all 6 of these mRNA, depending on the conditions, in cells and tumors of both mouse and human origin. Our data also showed that the lung adenocarcinoma xenograft tumors had more targets downregulated than the nonmalignant adenoma-containing lungs from the miR-31/CCSP mice, which is consistent with the more aggressive, late-stage lung adenocarcinomas expressing more miR-31.

In addition, we determined that miR-31 targeting of the negative regulators of RAS/MAPK signaling was sufficient to increase ERK activation, which markedly contributes to cellular transformation and proliferation. The modest increase in basal ERK activation in nonmalignant lung cells overexpressing miR-31 is consistent with findings in previous reports that moderate activation of the RAS/MAPK pathway is advantageous for transformation, whereas high levels of ERK signaling lead to cell-cycle arrest (44-46). Furthermore, this increase in basal ERK activity, and not a transient spike in ERK activation, has been shown to promote cycle entry by allowing for the accumulation of proteins required for cell-cycle entry, while at the same time repressing the expression of genes that inhibit proliferation (47). We show that expression of each of the negative regulators of RAS/MAPK signaling, except SPRY3, inhibited the increased proliferation induced with elevated levels of miR-31, suggesting that downregulation of 5 of these proteins by miR-31 contributes to miR-31-induced proliferation. Moreover, inhibition of MEK was reported to reduce lung adenocarcinoma proliferation induced by miR-31, but the mechanism for this was unknown (22). We showed that MEK inhibition suppressed miR-31-induced proliferation of nonmalignant lung epithelial cells. With increased RAS/MAPK pathway signaling in $76 \%$ of lung adenocarcinomas and mutations in $R A S$ genes and $B R A F$ only able to explain half of these (8), there must be other mechanisms of RAS/MAPK pathway activation. Our data provide a mechanism, involving miR-31, for the regulation of 6 mRNA that negatively control the RAS/MAPK pathway in the lung, leading to increased RAS/MAPK pathway activation. These results also provide an impetus to further investigate these genes in RAS/MAPK signaling in lung cancer.

\section{Methods}

Cell culture, transfections, reagents, and retroviral production and infection. All lung cell lines were cultured in RPMI 1640 supplemented with $10 \%$ FBS, L-glutamine, and penicillin/streptomycin and were provided by Pierre Massion, William Pao, and Jin Chen of Vanderbilt University (Nashville, Tennessee, USA) or David Jones of Memorial Sloan Kettering (New York City, New York, USA). NIH3T3 and 293T cells (both from ATCC) were cultured in DMEM supplemented with 10\% FBS and penicillin/streptomycin. Transfection of miR mimic, control RNA, inhibitor, or control inhibitor was done using DharmaFECT 1 (all from GE Healthcare). Plasmids were transfected using Lipofectamine 2000 (Thermo Fisher) as per the manufacturer's protocol. Six hours following transfection, transfection media were replaced with culture media. In addition to transfecting miR-31 mimic into cells, miR-31 was also overexpressed in cells using pBabe (puromycin selection marker) retroviral or lentiviral (GFP expression) vectors. For both pBabe and lentiviral and retroviral production, vectors were transfected into 293T Phoenix cells and virus was collected. Lung cells were infected with retrovirus containing media with $8 \mu \mathrm{g} / \mathrm{ml}$ polybrene. pBabe-infected cells were selected with puromycin for 7 days, and stable pools were used for experiments. Lentivirus-infected cells were evaluated for GFP expression and only used if $>90 \%$ of the cells were GFP positive. TaqMan qRT-PCR (see below) was used to verify miR-31 expression in all cells used for these studies. MEK inhibitors selumetinib (AZD6244) and trametinib (GSK1120212) were purchased from Selleckchem. All cDNAs were purchased from GE Healthcare with the exception of SPRY1, which was obtained from Addgene.

Patient samples. Deidentified frozen samples (non-small-cell lung adenocarcinoma and normal lung) collected from 2002 to 2013 were obtained from the Vanderbilt University Medical Center Lung Biorepository, which banked samples following patient consent. All samples were from surgical resections and were evaluated by a board-certified pathologist. All tumor samples used were determined to be $>80 \%$ tumor by evaluation of $\mathrm{H} \& \mathrm{E}$-stained sections. H\&E-stained sections of each normal sample were evaluated and determined to lack any precancerous lesions. Clinicopathologic features are listed in Supplemental Table 1. Another cohort of patients with lung adenocarcinoma was obtained from the TCGA database. 
Cell proliferation and transformation assays. For MTT assays, cells $(5,000$ per well) were placed in 96-well plates; the following day, cells were transfected with $50 \mathrm{nM}$ miR-31 mimic, inhibitor, or a corresponding control (all from GE Healthcare). The 0-hour time point was measured at the time of transfection. MTT assays were performed every 24 hours after transfection for 3 days as we previously reported (48), and absorbance was measured on an Emax Precision Microplate Reader (Molecular Devices). Cell viability was assessed by trypan blue dye exclusion. Clonogenic and soft agar assays were conducted as previously reported (48). Briefly, for clonogenic assays, cells infected with empty pBabe or pBabe retrovirus encoding miR-31 were placed in 6-well plates at low density (500 cells per well). After 14 days, cells were fixed with methanol, stained with crystal violet, and counted. For the soft agar assays, the pBabe-infected cells were suspended in $0.6 \%$ agarose and placed in 6-well plates $\left(10^{4}\right.$ cells per well) and processed identically to cells in the clonogenic assays. For the experiments using MEK inhibitors, 3,000 cells per well were placed in 96-well plates and transfected with either miR-31 mimic or RNA control the next day. Six hours later, the transfection media were removed and replaced with media containing either $100 \mathrm{nM}$ MEK inhibitor or DMSO control, and MTT assays performed. Experiments in which miR-31 targets were expressed were performed using $\mathrm{H} 1993$ cells that had been infected with a miR-31-expressing retrovirus or empty retrovirus control. Cells were then transfected with a vector encoding the cDNA of individual miR-31 targets or vector control, and MTT assays were performed.

Mice. FVB tet-O-mmu-miR-31 transgenic mice were generated using the pTRE2 vector (Clontech) and standard transgene methodology (Supplemental Figure 3) and were intercrossed with FVB CCSP-rtTA (49) and FVB tet-O-KRas ${ }^{G 12 D}$ (50) mice to obtain the mice needed for this study. All transgenic mice used were hemizygous, and both male and female mice and littermate controls were used in all analyses. Mice were administered either control drinking water ( $5 \%$ sucrose) or dox-containing drinking water ( $0.5 \mathrm{~g} / \mathrm{l} \mathrm{dox}, 5 \%$ sucrose $)$, starting at 4 to 6 weeks of age until sacrifice. For xenograft studies, 5- to 6-week-old athymic nude mice (Harlan) were injected subcutaneously in the flanks with $5 \times 10^{5} \mathrm{H} 1993$ cells or $1 \times 10^{6} \mathrm{H} 1437$ or $\mathrm{H} 460$ cells infected with empty pBabe retrovirus or pBabe encoding miR-31. Tumor volume was calculated from measurements with electronic calipers. At time of sacrifice $(22,24$, and 18 days, respectively, after injections), tumors were removed, weighed, and frozen for further analysis. For urethane chemical carcinogenesis, 6-week-old FVB miR-31/CCSP mice and single-transgenic CCSP control littermates were given a single intraperitoneal injection of urethane $(1 \mathrm{~g} / \mathrm{kg})$ and then administered water with dox for 6 weeks.

$q R T-P C R$. RNA was isolated using TRIzol (Thermo Fisher) according to the manufacturer's protocol. cDNA synthesis and quantification was described previously (48). SYBR Green (Qiagen) was used to quantify cDNA generated from mRNA and TaqMan miR assays (Thermo Fisher) for miR. Levels of $\beta$-actin were used to normalize mRNA in mouse and human tissue. For miR, snoRNA-202 was the endogenous control in mouse tissues and RNU6B was the endogenous control in human tissues. Assays were performed in triplicate, and primer sequences are listed in the Supplemental Methods. For mRNA analysis following transfection of miR-31 mimic, miR-31 inhibitor, or controls, cells were harvested 48 hours after transfection.

Western blotting. Whole-cell lysates from human cell lines and mouse tissues were generated and Western blotted as previously described (51). Antibodies against RASA1 (B4F8, Santa Cruz), SPRED1
(PA5-20617, Thermo Fisher), SPRED2 (S7320, Sigma-Aldrich), SPRY1 (D9V6P, Cell Signaling), SPRY4 (ab59785, Abcam), pERK (9101, Cell Signaling), ERK (9102, Cell Signaling), and $\alpha$-tubulin (T6074, SigmaAldrich) were used. Densitometry for quantification of protein bands was performed using ImageJ software (http://imagej.nih.gov/ij/). For all miR-31 target genes, band intensities are relative to $\alpha$-tubulin. For phospho-ERK1/2, quantification is relative to total ERK1/2.

Histology/pathology. The left lobe of the lung was collected from mice at intervals and flash frozen for RNA and protein analysis. The remaining lobes were perfused with PBS, inflated with $10 \%$ formalin, and fixed for 48 hours. For the analysis of surface lung nodules, lungs were fixed in Bouin's fixative for 24 hours and scored on a dissecting microscope. Lungs were then embedded in paraffin, sectioned $(5 \mu \mathrm{m})$, H\&E stained, and evaluated by a board-vertified veterinary pathologist (K.L. Boyd). A detailed description of how the tumors were pathologically evaluated is in the Supplemental Methods. Total lung and tumor areas were determined using ImageJ software. Total tumor burden was calculated as the sum of the total tumor area divided by the total lung area per $\mathrm{H} \& \mathrm{E}$-stained section of lung. Although lung tumors were mostly symmetrical, two diameter measurements per H\&E-stained section were evaluated using MetaMorph software (Molecular Devices) and represented as mean diameter per tumor. Ki67 IHC was performed by the Vanderbilt Translational Pathology Shared Resource with a standard protocol. Quantification of Ki67-positive cells from at least 5 separate fields per section was done using Image (module used can be found at the following web address: http://uab.mathbiol.org/ki67).

Intracellular flow cytometry. Beas-2B cells transfected with miR31 mimic or RNA control were incubated in serum-free medium for 72 hours. Serum (10\%) was added, and, at intervals, the cells were harvested by trypsinization and fixed with $1.5 \%$ paraformaldehyde. Following incubation with cold methanol overnight and rehydration, cells were incubated with anti-phospho-ERK1/2 (Thr202/Tyr204; 9101, Cell Signaling) or anti-ERK1/2 (9102, CST), washed, incubated with Alexa Fluor 647-conjugated anti-rabbit IgG (4414, Cell Signaling), and analyzed by flow cytometry (BD Fortessa). Normalized mean fluorescence intensity (MFI) was calculated by dividing the MFI of cells with anti-phospho-ERK by the MFI of cells with anti-total ERK and multiplying by $1 \times 10^{4}$.

Luciferase assays. Genomic DNA from human lung epithelial cells was used to clone the entire 3'-UTR or, if too long, the part of the 3'-UTR sequence that we determined to have potential miR-31-binding sites of LATS2, RASA1, SPRED1, SPRED2, SPRY1, SPRY3, and SPRY4 into a pmiRGLO Luciferase miR Target Expression Vector (Promega). Two separate pieces of the 3 '-UTR for SPRED1 were cloned into different vectors. Luciferase assays were conducted as previously described (52).

Analysis of TCGA lung adenocarcinoma data. miR expression profiles for lung adenocarcinoma and normal lung tissue samples were obtained from the TCGA data portal (https://tcga-data.nci.nih.gov/ tcga/) in May 2014 (for Figure 1, D-G) and December 2014 (for Figure 9C). miR expression, normalized by reads per million, were $\log _{2}$ transformed prior to evaluation. A detailed description of the analyses is provided in the Supplemental Methods.

Statistics. Unless otherwise stated, values represent mean \pm SEM, and 1-tailed Student's $t$ test was used for comparisons. Spearman's rank correlation determined the association between miR-31 and target mRNA expression (Figure 9B and Supplemental Table 3). $P$ values of less than 0.05 were considered significant. Statistics for the TCGA data are described in the Supplemental Methods. 
Study approval. All experiments involving mice were approved by the Vanderbilt Institutional Animal Care and Use Committee and followed all state and federal rules and regulations. All human samples used in experiments were deidentified and obtained from the Vanderbilt University Medical Center Lung Biorepository, which banked samples following informed consent.

\section{Author contributions}

MDE and CME designed the research studies. MDE, TM, RD, and MPA conducted experiments. RM, XC, and ZZ analyzed TCGA data. TA made the tet-o-miR-31 mice and provided advice. TSB provided mice, reagents, and advice. KLB performed the histolog$\mathrm{ical} /$ pathological analysis. MDE, CME, and KLB wrote the manuscript. All authors edited the manuscript.

\section{Acknowledgments}

We thank Rosana Eisenberg and David Carbone of the Vanderbilt University Medical Center Lung Biorepository for human samples; Pierre Massion, William Pao, Jin Chen, and David Jones for cell lines; and Jamie Ausborn and Taylor Sherrill for technical assistance. This work was supported by the American Cancer Society-Kirby Foundations Fund Postdoctoral Fellowship PF-13088-01-CSM (to M.D. Edmonds), National Cancer Institute (NCI) grants R01CA177786 (to C.M. Eischen) and R03CA167695 (to Z. Zhao), the Vanderbilt-Ingram Cancer Center Thoracic Program, Clinical and Translational Science Award UL1TR000445 from the National Center for Advancing Translational Sciences, NCI P50CA090949 SPORE in Lung Cancer, and NCI Cancer Center Support Grant P30CA068485, utilizing the Vanderbilt Translation Pathology Shared Resource.

Address correspondence to: Christine M. Eischen, Vanderbilt University Medical Center, Department of Pathology, Microbiology and Immunology, MCN C3321, 1161 21st Avenue South, Nashville, Tennessee 37232-2561, USA. Phone: 615.322.3234; E-mail: christine.eischen@vanderbilt.edu.
1. Siegel RL, Miller KD, Jemal A. Cancer statistics, 2015. CA Cancer J Clin. 2015;65(1):5-29.

2. Vidigal JA, Ventura A. The biological functions of miRNAs: lessons from in vivo studies. Trends Cell Biol. 2015;25(3):137-147.

3. Costinean S, et al. Pre-B cell proliferation and lymphoblastic leukemia/high-grade lymphoma in E(mu)-miR155 transgenic mice. Proc Natl Acad Sci U S A. 2006;103(18):7024-7029.

4. Medina PP, Nolde M, Slack FJ. OncomiR addiction in an in vivo model of microRNA-21induced pre-B-cell lymphoma. Nature. 2010;467(7311):86-90.

5. Sandhu SK, et al. B-cell malignancies in microRNA Emu-miR-17 92 transgenic mice. Proc Natl Acad Sci U S A. 2013;110(45):18208-18213.

6. Hatley ME, et al. Modulation of K-Ras-dependent lung tumorigenesis by MicroRNA-21. Cancer Cell. 2010;18(3):282-293.

7. Riely GJ, Marks J, Pao W. KRAS mutations in non-small cell lung cancer. Proc Am Thorac Soc. 2009;6(2):201-205

8. Meyerson M. Comprehensive molecular profiling of lung adenocarcinoma. Nature. 2014;511(7511):543-550.

9. Creighton CJ, et al. Molecular profiling uncovers a p53-associated role for microRNA-31 in inhibiting the proliferation of serous ovarian carcinomas and other cancers. Cancer Res. 2010;70(5):1906-1915.

10. Greenberg E, et al. Regulation of cancer aggressive features in melanoma cells by microRNAs. PLoS One. 2011;6(4):e18936.

11. Guo J, et al. Differential expression of microRNA species in human gastric cancer versus non -tumorous tissues. J Gastroenterol Hepatol. 2009;24(4):652-657.

12. Kim HS, et al. MicroRNA-31 functions as a tumor suppressor by regulating cell cycle epithelialmesenchymal transition regulatory proteins in liver cancer. Oncotarget. 2015;6(10):8089-8102.

13. Laurila EM, Kallioniemi A. The diverse role of miR-31 in regulating cancer associated phenotypes. Genes Chromosomes Cancer.
2013;52(12):1103-1113.

14. Schaefer A, et al. Diagnostic and prognostic implications of microRNA profiling in prostate carcinoma. Int J Cancer. 2010;126(5):1166-1176.

15. Wszolek MF, et al. A MicroRNA expression profile defining the invasive bladder tumor phenotype. Urol Oncol. 2011;29(6):794-801 e1.

16. Yan LX, et al. MicroRNA miR-21 overexpression in human breast cancer is associated with advanced clinical stage, lymph node metastasis and patient poor prognosis. RNA. 2008;14(11):2348-2360.

17. Zhang $\mathrm{T}$, et al. The oncogenetic role of microRNA-31 as a potential biomarker in oesophageal squamous cell carcinoma. Clin Sci (Lond). 2011;121(10):437-447.

18. Tan X, et al. A 5-microRNA signature for lung squamous cell carcinoma diagnosis and hsa-miR-31 for prognosis. Clin Cancer Res. 2011;17(21):6802-6811.

19. Slattery ML, et al. An evaluation replication of miRNAs with disease stage colorectal cancer-specific mortality. Int J Cancer. 2014;137(2):428-438.

20. Liu CJ, et al. miR-31 ablates expression of the HIF regulatory factor FIH to activate the HIF pathway in head and neck carcinoma. Cancer Res. 2010;70(4):1635-1644.

21. Liu X, et al. MicroRNA-31 functions as an oncogenic microRNA in mouse and human lung cancer cells by repressing specific tumor suppressors. J Clin Invest. 2010;120(4):1298-1309.

22. Meng W, et al. MicroRNA-31 predicts the presence of lymph node metastases and survival in patients with lung adenocarcinoma. Clin Cancer Res. 2013;19(19):5423-5433.

23. Mahler JF, Stokes W, Mann PC, Takaoka M, Maronpot RR. Spontaneous lesions in aging FVB/N mice. Toxicol Pathol. 1996;24(6):710-716

24. Westcott PM, et al. The mutational landscapes of genetic and chemical models of Kras-driven lung cancer. Nature. 2015;517(7535):489-492

25. Mori I, et al. Aberrant expression of cyclin D1 in pulmonary proliferative lesions induced by high doses of urethane in transgenic mice carrying the human prototype c-H-ras gene. J Vet Med Sci. 2001;63(3):261-268.

26. Huang W, Sherman BT, Lempicki RA. Systematic and integrative analysis of large gene lists using DAVID bioinformatics resources. Nat Protoc. 2009;4(1):44-57.

27. Huang W, Sherman BT, Lempicki RA. Bioinformatics enrichment tools: paths toward the comprehensive functional analysis of large gene lists. Nucleic Acids Res. 2009;37(1):1-13.

28. Yang MH, et al. Elevated microRNA-31 expression regulates colorectal cancer progression by repressing its target gene SATB2. PLoS One. 2013;8(12):e85353.

29. Clark GJ, Westwick JK, Der CJ. p120 GAP modulates Ras activation of Jun kinases and transformation. J Biol Chem. 1997;272(3):1677-1681.

30. Bundschu K, Walter U, Schuh K. The VASPSpred-Sprouty domain puzzle. J Biol Chem. 2006;281(48):36477-36481.

31. Samatar AA, Poulikakos PI. Targeting RAS-ERK signalling in cancer: promises and challenges. Nat Rev Drug Discov. 2014;13(12):928-942.

32. Dankort D, Filenova E, Collado M, Serrano M, Jones K, McMahon M. A new mouse model to explore the initiation, progression, and therapy of BRAFV600E-induced lung tumors. Genes Dev. 2007;21(4):379-384.

33. Saxena M, Christofori G. Rebuilding cancer metastasis in the mouse. Mol Oncol. 2013;7(2):283-296.

34. Wang S, et al. Down-regulated expression of SATB2 is associated with metastasis and poor prognosis in colorectal cancer. J Pathol. 2009;219(1):114-122.

35. Koumangoye RB, et al. SOX4 interacts with EZH2 and HDAC3 to suppress microRNA-31 in invasive esophageal cancer cells. Mol Cancer. 2015;14(1):24.

36. Zhang Q, Padi SK, Tindall DJ, Guo B. Polycomb protein EZH2 suppresses apoptosis by silencing the proapoptotic miR-31. Cell Death Dis. 2014;5:e1486.

37. Garzon R, Marcucci G, Croce CM. Target- 
ing microRNAs in cancer: rationale, strategies and challenges. Nat Rev Drug Discov. 2010;9(10):775-789.

38. Ebert MS, Sharp PA. Roles for microRNAs in conferring robustness to biological processes. Cell. 2012;149(3):515-524.

39. Hu C, Huang F, Deng G, Nie W, Huang W, Zeng X. miR-31 promotes oncogenesis in intrahepatic cholangiocarcinoma cells via the direct suppression of RASA1. Exp Ther Med. 2013;6(5):1265-1270.

40. Sun D, et al. MicroRNA-31 activates the RAS pathway and functions as an oncogenic MicroRNA in human colorectal cancer by repressing RAS p 21 GTPase activating protein 1 (RASA1). J Biol Chem. 2013;288(13):9508-9518.

41. Shaw AT, et al. Sprouty-2 regulates oncogenic $\mathrm{K}$-ras in lung development and tumorigenesis. Genes Dev. 2007;21(6):694-707.

42. Tennis MA, Van Scoyk MM, Freeman SV, Vandervest KM, Nemenoff RA, Winn RA. Sprouty-4 inhibits transformed cell growth, migration and invasion, and epithelial-mesenchymal transi- tion, and is regulated by Wnt7A through PPAR $\gamma$ in non-small cell lung cancer. Mol Cancer Res. 2010;8(6):833-843.

43. Ledda F, Paratcha G. Negative regulation of Receptor Tyrosine Kinase (RTK) signaling: a developing field. Biomark Insights. 2007;2:45-58.

44. Sewing A, Wiseman B, Lloyd AC, Land H. Highintensity Raf signal causes cell cycle arrest mediated by p21Cip1. Mol Cell Biol.1997;17(9):5588-5597.

45. Woods D, Parry D, Cherwinski H, Bosch E, Lees E, McMahon M. Raf-induced proliferation or cell cycle arrest is determined by the level of Raf activity with arrest mediated by p21Cip1. Mol Cell Biol. 1997;17(9):5598-5611.

46. MiRza AM, Gysin S, Malek N, Nakayama K, Roberts JM, McMahon M. Cooperative regulation of the cell division cycle by the protein kinases RAF and AKT. Mol Cell Biol. 2004;24(24):10868-10881.

47. Yamamoto T, Ebisuya M, Ashida F, Okamoto K, Yonehara S, Nishida E. Continuous ERK activation downregulates antiproliferative genes throughout G1 phase to allow cell-cycle progres- sion. Curr Biol. 2006;16(12):1171-1182.

48. Grieb BC, et al. Oncogenic protein MTBP interacts with MYC to promote tumorigenesis. Cancer Res. 2014;74(13):3591-3602.

49. Tichelaar JW, Lu W, Whitsett JA. Conditional expression of fibroblast growth factor-7 in the developing and mature lung.J Biol Chem. 2000;275(16):11858-11864.

50. Fisher GH, et al. Induction and apoptotic regression of lung adenocarcinomas by regulation of a K-Ras transgene in the presence and absence of tumor suppressor genes. Genes Dev. 2001;15(24):3249-3262.

51. Alt JR, Bouska A, Fernandez MR, Cerny RL, Xiao $\mathrm{H}$, Eischen CM. Mdm2 binds to Nbs1 at sites of DNA damage and regulates double strand break repair. J Biol Chem. 2005;280(19):18771-18781.

52. McGirt LY, Adams CM, Baerenwald DA, Zwerner JP, Zic JA, Eischen CM. miR-223 regulates cell growth and targets proto-oncogenes in mycosis fungoides/cutaneous T-cell lymphoma. J Invest Dermatol. 2014;134(4):1101-1107. 